\title{
A Two-Phase Model for the Mechanical Behavior of Semicrystalline Polymers
}

\author{
L. Cangémi ${ }^{1}$ and Y. Meimon ${ }^{1}$ \\ 1 Institut français du pétrole, 1 et 4, avenue de Bois-Préau, 92852 Rueil-Malmaison Cedex - France \\ laurent.cangemi@ifp.fr - yves.meimon@ifp.fr
}

\begin{abstract}
Résumé - Un modèle biphasique pour le comportement mécanique des polymères semi-cristallins - L'utilisation croissante des polymères semi-cristallins (PSC) dans des structures industrielles complexes nécessite la mise au point de modèles de comportement mécanique fiables et prédictifs. Après avoir rappelé les différentes approches développées dans la littérature, nous présentons des résultats expérimentaux obtenus sur du PVDF et sur du PA11. Ceux-ci montrent que ces polymères sont sensibles à la contrainte moyenne et que leur déformation volumique, loin d'être négligeable, doit être prise en compte pour la modélisation du comportement du matériau. À partir des modèles mécaniques existant dans la littérature, on peut définir trois grandes classes de modélisation en fonction de l'échelle de représentation utilisée (micro, méso et macro) et de la nature des informations qu'elles permettent d'extraire de la microstructure. Il s'avère que l'adoption d'une échelle mésoscopique intermédiaire constitue un bon compromis entre difficultés théoriques et richesse de modélisation. Ce choix pragmatique s'appuie sur une représentation biphasique particulière de la microstructure des PSC. Elle permet d'effectuer une transition continue entre l'échelle mésoscopique et l'échelle macroscopique. L'utilisation de la thermodynamique des systèmes ouverts conduit à introduire une équation de couplage mécanique entre une phase solide (comprenant le cristal) et une phase dite «molle » ou « fluide » (essentiellement amorphe). Le modèle est ensuite complété par une formulation élastoviscoplastique permettant de décrire la variation de volume particulière des PSC, leur sensibilité à la vitesse de sollicitation et à la contrainte moyenne. La modélisation est confrontée aux résultats d'essais de traction effectués sur ces deux types de PSC utilisés dans l'industrie pétrolière.
\end{abstract}

Mots-clés : polymères semi-cristallins, PA11, PVDF, essais de traction, essais de compression, fluage, relaxation, élastoviscoplasticité, thermodynamique des systèmes ouverts.

\begin{abstract}
A Two-Phase Model for the Mechanical Behavior of Semicrystalline Polymers - The increasing use of semicrystalline polymers (SCP) in complex industrial structures, requires the development of reliable and predictive mechanical behavior models. The various approaches developed in the literature are considered and experimental results obtained from tests on $\mathrm{PVF}_{2}$ and on PA11 are presented. These results show that these polymers are sensitive to the mean stress, and that the volume strain cannot be neglected and should be taken into account to model the material behavior. From the mechanical models existing in the literature, one can define three categories according to the scale of representation used (micro, meso and macro) and the information which they allow to extract from the microstructure. It turns out that the adoption of an intermediate meso-scale constitutes a good compromise between theoretical difficulties and richness of modeling. This pragmatic choice leans on a two-phase representation of SCP, who allows to make a continuous transition from the microscopic scale to the macroscopic scale. The thermodynamics of open systems leads to introduce a mechanical coupling
\end{abstract}


equation between a "solid" phase (including the crystal) and a "soft" phase or a "fluid" phase (essentially amorphous). The model is then completed by an elastoviscoplastic formulation allowing to describe the particular volume strain of SCP, the effects of the loading rate and of the mean stress. The model is validated against results of tension tests carried out on these two types of SCP used in the oil industry.

Keywords: semicrystalline polymers, PA11, PVF ${ }_{2}$, tensile tests, compressive tests, creep, relaxation, elastoviscoplasticity, thermodynamics of open systems.

\section{NOTATIONS}

\section{Polymers}

$\begin{array}{ll}\text { PA6 } & \text { Poly(amide 6) } \\ \text { PA11 } & \text { Poly(amide 11) } \\ \text { PBFP } & \text { Poly(bis-trifluoroethoxy phosphazene) } \\ \text { PC } & \text { Poly(carbonate) } \\ \text { PE } & \text { Poly(ethylene) } \\ \text { PEEK } & \text { Poly(etheretherketone) } \\ \text { PMMA } & \text { Poly(methyl methacrylate) } \\ \text { POM } & \text { Poly(oxymethylene) } \\ \text { PP } & \text { Poly(propylene) } \\ \text { PS } & \text { Poly(styrene) } \\ \text { PTFE } & \text { Poly(tetrafluorethylene) } \\ \text { PVC } & \text { Poly(vinylcarbonate) } \\ \text { PVF } 2 & \text { Poly(vinylidenefluoride) } \\ \text { PVF } & \text { Poly (vinyl formal). }\end{array}$

\section{Variables and parameters}

a Isotropic hardening parameter (dimensionless)

$B \quad$ Biot's coefficient (elastic coupling between the hard phase and the soft phase; dimensionless)

$b_{s} \quad$ Yield surface parameter (dimensionless)

$C_{s} \quad$ Cohesion $(\mathrm{Pa})$

$I_{1} \quad$ First invariant of the stress tensor $=\operatorname{tr}(\underline{\underline{\sigma}})$

$\begin{array}{ll}J_{2} & \text { Second invariant of the stress deviator }=\frac{1}{2} \underline{\underline{s}}: \underline{\underline{s}} \\ q & \text { Deviatoric stress }=\sqrt{3} J_{2}^{1 / 2}\end{array}$

$K_{s} \quad$ Parameter of the viscous law $(\mathrm{Pa})$

$m \quad$ Fluid mass movements $(\mathrm{kg})$

$M_{a} \quad$ Biot's modulus (coupling between the hard phase and the soft phase; $\mathrm{Pa}$ )

$P \quad$ Free amorphous pressure $(\mathrm{Pa})$

$P_{c r}^{0} \quad$ Initial pressure in the solid phase $(\mathrm{Pa})$

$T \quad$ Temperature (K)

\section{Greek symbols}

$\underline{\sigma} \quad$ Total Cauchy Stress tensor

$\underline{\underline{\sigma}}_{s} \quad$ Effective Cauchy Stress tensor (acting on solid phase)

$\underline{\underline{\varepsilon}} \quad$ Euler-Almansi strain tensor

$\triangleq \quad$ Green-Lagrange strain tensor

$\underline{\underline{s}} \quad$ Deviator stress tensor $=(\underline{\underline{\sigma}}-1 / 3 \operatorname{tr}(\underline{\underline{\sigma}}))$

$\begin{array}{ll}\underset{\pi}{\underline{\tau}} & \text { Piola-Kirchhoff stress tensor } \\ \varepsilon_{v p}^{\text {vol }} & \text { Plastic volume strain } \\ \varepsilon_{0}^{d} & \text { Initial plastic strain } \\ \alpha & \text { Plastic volume strain intensity (dimensionless) } \\ \beta & \begin{array}{l}\text { Fluid phase coefficient of plastic contraction or } \\ \text { dilatation (dimensionless) }\end{array} \\ \beta_{s} & \begin{array}{l}\text { Solid phase coefficient of plastic contraction } \\ \text { (dimensionless) }\end{array} \\ \eta & \text { Viscous parameter: relaxation time }\left(\mathrm{s}^{-1}\right) \\ \mu_{s} & \text { Internal friction parameter (dimensionless) } \\ \rho_{m} & \text { Density of the fluid phase } \\ \phi_{a} & \text { Index parameter of the free amorphous proportion } \\ & \text { (dimensionless). }\end{array}$

\section{Others}

\section{INTRODUCTION}

Initially developed for product packaging, polymers are more and more associated with various structural materials in complex structures as load-bearing components. Their introduction in the fields of the energy and of the transport (pipelines, fuel tanks for automobiles, pump bodies, water and gas supply pipes, etc.) involves thermomechanical loads acting in strongly aggressive environment. In the oil domain, polymers are mainly used as seal barriers in flexible metal pipes for oil transportation. They should resist to high pressures (until $100 \mathrm{MPa}$ ) of aggressive chemical fluids, at service temperatures ranging from $80^{\circ} \mathrm{C}$ to $140^{\circ} \mathrm{C}$. In such a context, semicrystalline polymers (SCP) are often selected with respect to their chemical inactivity and to their good mechanical behavior, for a large range of temperature. However, among the met problems, we can quote the very complex phenomenon of blistering which is a damage process due to explosive decompression in flexible pipes. Under the pipe service pressure, gas saturates the polymer. Designed for use over several decades, these structures are liable to sudden operating interruptions, resulting in a sharp drop in pressure in the pipes. The gas present in the polymer 
is forced to diffuse outside the material and could damage it [1]. Experiments on test tubes show that the nature and the importance of the damage depend on the processing technique and on numerous factors (mechanical loading, temperature, physicochemical couplings, etc.) [2]. This complexity of phenomena is also illustrated by the aging of materials. For example, the hydrolysis of PA11 results in a decrease of its mechanical properties and ends in a important embrittlement of the material. This one is due to a coupling of several physical processes: diffusion of water, loss of plasticizers, cuts of macromolecular chains. Hence, to design structures including SCP and to reasonably predict their life time, a mechanical model should reproduce these phenomena, and be inserted into a software using the finiteelement method (FEM).

The plastic behavior of SCP is intimately related to their two-phase microstructure, constituted by crystalline lamellae and by macromolecules in an amorphous state. Research efforts concerned generally the development of experimental techniques in order to understand the physical mechanisms of the plastic deformation of polymers [3-5]. Although an unified theory was not yet born, some elementary mechanisms were identified. They give an idea of the processes involved at several scales. Generally speaking, one admits that the successive stages of the deformation are the result of sliding of chains in the amorphous phase, interlamellar separation, mechanisms of crystallographic deformation and morphological evolutions at large strains. These mechanisms were reviewed by several authors [6, 7].

Some studies also tried to simulate and to predict the behavior of polymers in a wide range of deformation. So, considering amorphous polymers, the behavior at the scale of a "molecular unity" can be considered as representative of the global behavior of the material. In this case, the macroscopic behavior is directly deduced from an analysis at the macromolecule scale [8-12]. As regards the SCP, their complex and heterogeneous microstructure forbids this type of analysis. A synthesis of the approaches most usually used is proposed in [13]. Generally, the authors attempt to model the material behavior up to the large deformations. The models are essentially empirical and one-dimensional. They tempt to predict various aspects of the viscoelastic and viscoplastic behavior of thermoplastics by using combinations of simple mechanical models such as Voigt and Maxwell, or more sophisticated choices of the dissipation function [14]. A quantitative model based on the theory of the plastic waves was as well developed [15] from the observation that the plastic strain is often linked to a shear process, localized in bands more or less wide according to the type of material. Then, Orowan's Formula allows to define an explicit shape for the plastic propagation, similar to Eyring's formalism [16] and justified by experiments. On the basis of an analogy with the theory of the rubberlike elasticity [17], the effects of hardening at large strains are often introduced through a rubberlike entropic back stress. The stress is divided into an effective stress, thermally activated and an internal stress, the intensity of which is proportional to the elongation of a macromolecular pseudonetwork. Without being exhaustive, one will quote [18] and [19] for glassy polymers and, [14] and [20] for polymers in general.

Some approaches, stemming from the metal industry, describe the viscoplastic behavior of SCP. As for metals, the starting point is phenomenological and uses creep tests. By observing the strain rate behavior versus time, it is possible to decompose creeping into a primary and a secondary creep. The incapacity of Norton's law to describe all these phenomena brought the researchers to elaborate improved theories of the viscoplasticity. Let us quote, for example, the "Overstress" models initially introduced for metals at high temperature [21] and used to describe the viscoplastic behavior of polypropylene [22]. In that case, one supposes only the existence of an internal stress or "backstress" which evolves with the deformation. For this material, a more recent model [23], based on a notion of "stages of deformation", integrates a plastic yield and a nonlinear kinematical hardening into the plastic domain. These last two approaches can be integrated into a viscoplastic framework, the general concepts of which were unified by Perzyna [24] and Lemaitre and Chaboche [25].

Among the phenomenological approaches, we can mention the DNLR models (distribution of nonlinear relaxations) [26, 27], developed within the framework of irreversible thermodynamics for systems outside equilibrium. Here, the microstructural complexity is not described explicitly but approached by using a spectral analysis of the dissipation. This last one is introduced into a rigorous framework of thermodynamics and led to explicit choices of the relaxation spectra, the nonlinearity of the kinetics for the return to equilibrium and of the shape of the relaxed states.

Contrary to the previous approaches, micromechanical modeling was developed to bind the global macroscopic behavior to the crystallographic evolution of the material [2833]. Here, the mechanical behavior of a homogeneous material equivalent to the micro-heterogeneous material is deduced from the descriptions of the microstructure and the local behavior [34]. The choice of the elementary representative volume (ERV) at the microscopic scale (spherulite, crystalline lamellae, etc.) depends on the studied phenomena (damage, anisotropy, etc.). The autocoherent scheme supplies an expression of the effective properties in the case of a heterogeneous system, constituted by spherical heterogeneities randomly distributed in the volume. These models predict the specific effects of hardening arising at large strains but neglect certain aspects of the plastic behavior at small and average strains (sensibility to pressure, effects of volume changes, etc.). On a more fundamental level, theoretical difficulties also appear. For example, it is 
more difficult to take into account in this way, the effect of a peculiar topological distribution of spherulites. Besides, the viscoelastic nature of the material infers a coupling between space and time, that is an effect of long memory, which it is not easy to handle in the formalism [28, 35, 36]. So, this promising approach should be deepened for SCP before being applied to the analysis of structures by the classic FEM.

In this paper, a pragmatic approach (already presented in [1] and [37]), based both on experimental observations and on a particular interpretation of the microstructure, is presented. Section 1 redraws the experimental evidences which incite us to make a parallel with geomechanics. Certain peculiarities of the SCP mechanical behavior are analyzed from literature results and results of more specific tests carried out at the Institut français du pétrole (IFP).

In section 2, a two-phase interpretation of the microstructure is presented. It is based on physicochemical characterizations and on mechanical and rheological considerations. This interpretation at the mesoscopic scale allows to make a heuristic scaling towards the continuum mechanics, by taking place in the general framework of thermodynamics of open systems [38-42]. On the other hand, this choice is supported by the importance of exchanges phenomena of SCP with their industrial environment. Section 3 specifies the choice of the thermodynamic potential which allows to justify a coupling equation between both phases of the material. Section 4 introduces the viscoplastic formalism chosen to model the irreversible effects at the origin of the nonelastic strain of SCP and Section 5 presents a validation of the model for $\mathrm{PVF}_{2}$ and PA11.

\section{EXPERIMENTAL EVIDENCES}

\subsection{Domains of the Mechanical Behavior}

The mechanical behavior of SCP is strongly nonlinear and sensitive to physical and mechanical parameters such as temperature, rate effects, paths of loading, etc., so that it is difficult to reduce it to a precise and particular rheological behavior (elastic, viscoelastic, plastic, etc.). We prefer, at first, to speak about domains of behavior, which correspond to various stages of the material deformation [43]. These domains can be analyzed by using uniaxial tests. They are characterized by the loss of linearity of the stress-strain behavior and by the deformation recovery of the sample during a cycle of loading (capacity of the material to come back its initial state). The loss of linearity defines a threshold in stress and strain marking the entrance of the material into a viscoelastic or anelastic domain. This threshold, rather weak for most of the polymers, defines an initial linear elastic limited zone (of about $1 \%$ of strain). In the viscoelastic domain (associated to deformations of about 5\%), the recovery of strain, after a cycle closed in stress, is total (or quasi-total). Beyond, the deformation of the material is not completely recoverable. This irreversible behavior can reasonably be qualified as plastic or more exactly as viscoplastic. Then, a general formalism of constitutive equations with internal variables, introduced mainly for metals within the continuum framework of thermodynamics, allows to approach these various domains in a unified way. This general approach, applied to polymers, qualitatively reproduces the various aspects of their mechanical deformation: nonlinearity, rate sensitivity, creeping, complete or partial recovery [44]. The main difficulties for polymers lie in the big variety of mechanical responses (knee or hook of stress in tensile test, hardening at large strains (Fig. 1) and in the strong temporal nonlinearity of the mechanical behavior (strong rate sensitivity under short time loading; creep and relaxation under long time loading).

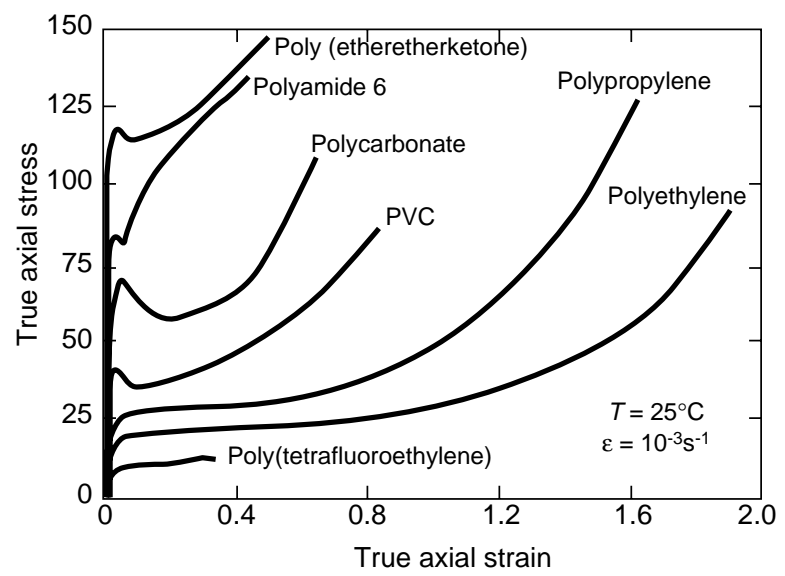

Figure 1

Plastic behavior in tension for a selection of amorphous and semicrystalline polymers (PEEK, PA6, PC, PVC, PP, PE, PTFE) (after [45]).

Through this paper, we analyze specificity of SCP mechanical behavior for true strains ranging from some percent to fifty percent. Hence, hardening effects due to the crystalline orientation and to the network stretching are not important and will be neglected. We assume that this hypothesis of small and average strains is sufficient to build a constitutive model useful for the analysis of industrial structures. Conversely, in this range of deformation, the developed model should be enough accurate, reliable and predictive. When structures are designed, the applied loading result locally in complex and multiaxial loading paths. This emphasizes the necessity to study the influence of variables such as the mean stress or the volumetric strain on the mechanical behavior of SCP. 


\subsection{Effect of the Mean Stress}

It is well known that the plastic behavior of polymers is strongly dependent on surrounding conditions of pressure. Studies quantified the effect of the hydrostatic pressure on various aspects of the mechanical behavior of PTFE and linear PE [46] as well as POM [47]. Tensile tests indicate that the hydrostatic pressure acts to increase Young's modulus and the yield stress. A pressure increase induces a pronounced hardening of the material. As regards the ductility or the fracture properties, the characteristics can vary from a material to the other one. For example, a pressure increase causes a decrease of the strain at failure for PTFE and linear PE [46] but an increase of this strain for POM (Fig. 2). From the POM study, it is suggested [47] that the yield stress pressure sensibility should be connected with the particular microstructural spherulitic morphology of SCP. These remarks show that there is a lot of interest to develop a mechanical model on the basis of a multi-scale approach. Section 2 will give a two-phase interpretation of the microstructure of SCP, taking in account these considerations.

Figure 2 shows that the yield stress of polymers is sensitive to the mean stress (or the first invariant of the stress tensor). The yield stress depends also on the main direction of loading. Significant variations of the yield stress were found in axial compressive tests, axial tensile tests, simple shear tests and plane compression tests performed on three glassy polymers [48]. Few studies exist on the multiaxial behavior of SCP. For example, tensile, torsion and compressive tests were performed on $\mathrm{PVF}_{2}$ tubes [49]. They showed that the yield stress increases slightly when comparing tensile and torsion results, but increases considerably (of 40\%) in compression (Fig. 3). These results show clearly that the mean stress is an important variable for analyzing plastic behavior of SCP.

\subsection{Mean Stress and Volume Change}

Most of the previous studies describe the influence of the hydrostatic pressure (or the mean stress) on polymers behavior without considering the evolution of the volumetric strain. However, for other materials like soils or granular materials, numerous studies show that this dependence should be connected with the volume change of the material. Thermodynamically, this variable is in duality with the mean stress. Hence, the effect of the confining pressure is directly linked to the compressibility and to the volume strain of the material. In the literature on polymers, this idea is rarely deepened and exploited. Nevertheless some authors considered this aspect of polymers behavior through experimental observations. In 1967, in a precursor work being inspired by knowledge acquired in soil mechanics, Whitney and Andrews were interested in the evolution of the volume change of some polymers [50]. By means of a mercury dilatometer, they measured the volume change which accompanies the elastic and plastic deformation of four glassy polymers (PMMA, PS, PC and PVF) during uniaxial compression. In the stage preceding the plastic flow, it was possible to measure a contraction of the material. They quantified the gap between a purely elastic prediction and the

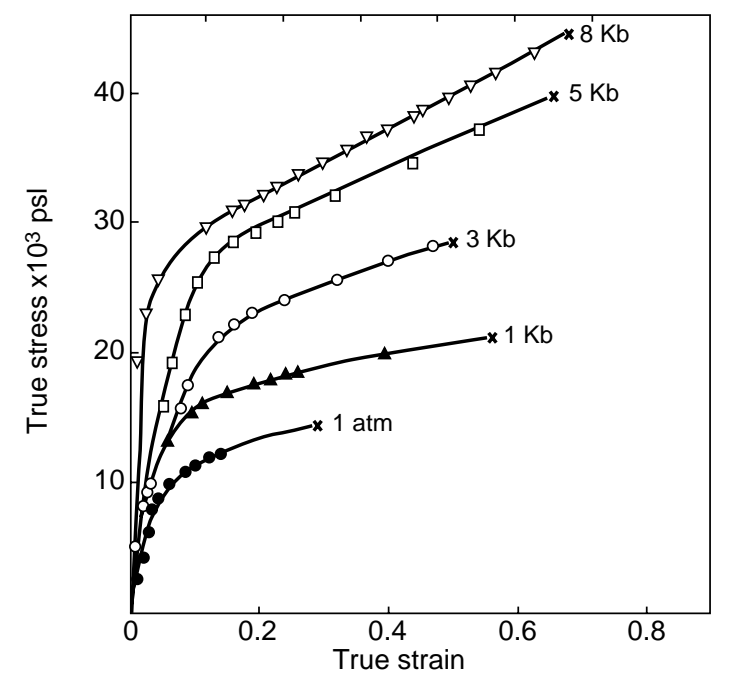

Figure 2

Tensile stress/strains curves for POM, for several hydrostatic pressures. After [47].

Reprinted from "Polymer Engineering and Science", with permission from Society of Plastics Engineers.

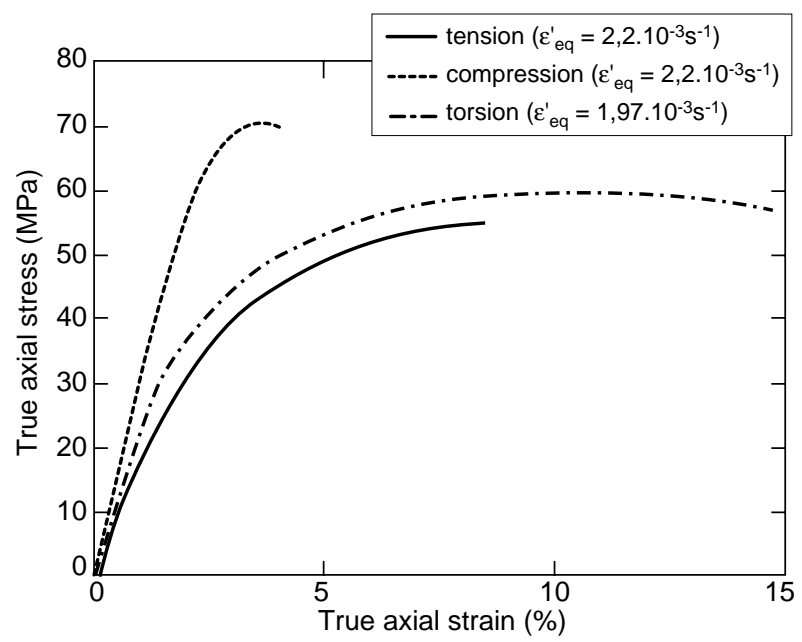

Figure 3

Tensile, torsion and compression true stress/true strains curves for $\mathrm{PVF}_{2}$ (after [47]). 

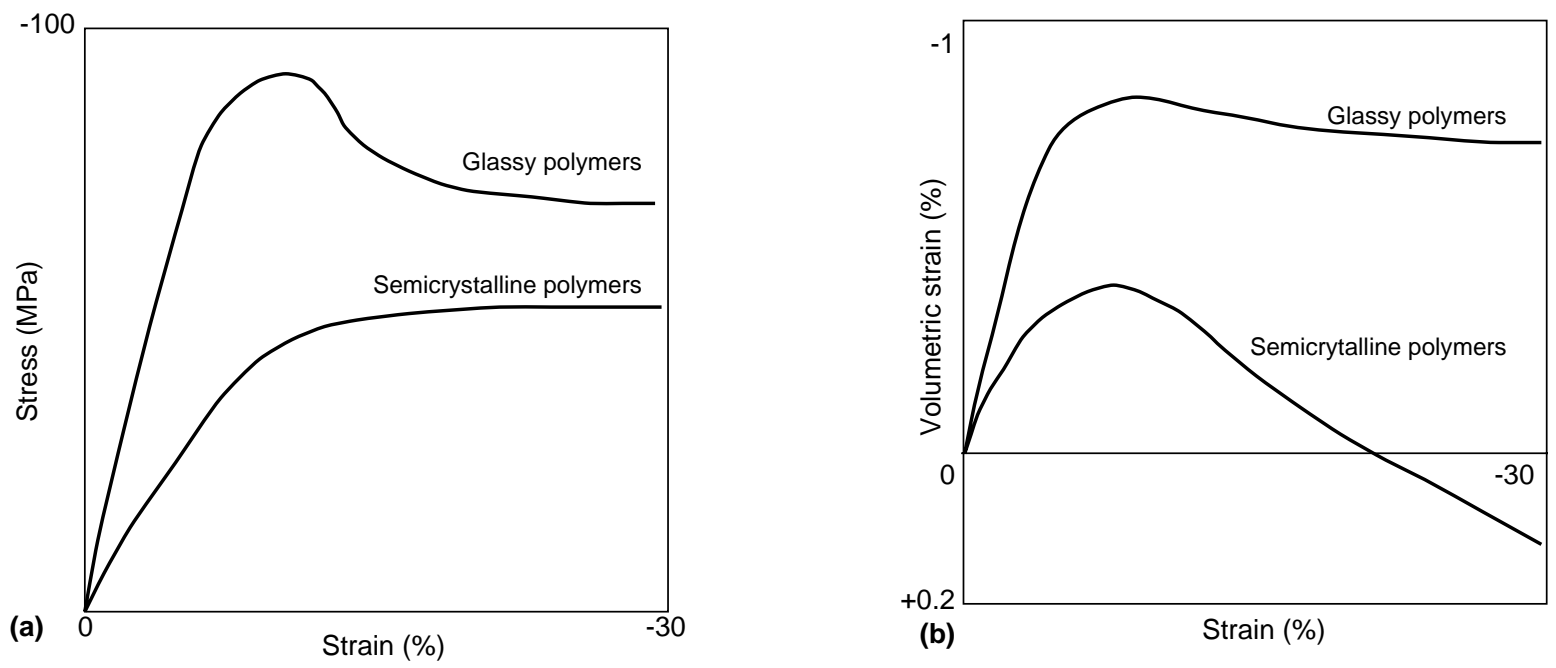

Figure 4

Schematic representation of stress/strain curves (a) and volume strain/axial strain (b) in compression on various polymers.

total volume strain, and called it the "volume plastic expansion". However, one should be cautious not to pronounce too fast on the sign (contraction or expansion) and on the nature of this quantity. Indeed, nonlinearity of the mechanical behavior of polymers brings in a complicated way phenomena of different nature: elastic contraction, plastic or viscoelastic contraction or expansion, etc. Only a fine analysis or a loading-unloading test would allow to discriminate between the various aspects of the nonelastic volume strain. A more recent study [51] concerns the volume strain of a wider range of polymers, including SCP. These experiments, comparable to those of Whitney and Andrews, show the existence of a plastic dilation in compression, relatively weak in the case of amorphous glassy polymers but very pronounced for SCP.

During the initial phase of the tests, a contraction due to the elasticity of materials is obtained. For SCP, when the compression stress exceeds the elastic domain, the sign of the volume strain rate changes and a volume expansion appears and grows considerably (Fig. 4). For PE and PP, the volume strain can even become positive. These results put sharply in evidence the existence of a plastic expansion in compression, denoted $\varepsilon_{v}^{p}$ and quantified by:

$$
\varepsilon_{v}^{p}=\varepsilon_{v}-\varepsilon_{v}^{e}=\varepsilon_{v}-\sigma_{m} / K
$$

where $\varepsilon_{v}$ is the total volume strain, $\varepsilon_{v}^{e}$ is the elastic volume strain and $K$ is the instantaneous elastic bulk modulus. The schematic evolution of the plastic volume strain versus the axial strain is given in the Figure 5, for both kinds of polymer. For glassy amorphous, the plastic volume strain is rather contracting, even if a slight tendency to dilation is observed on PVC (see [51]). Conversely, the three SCP tested

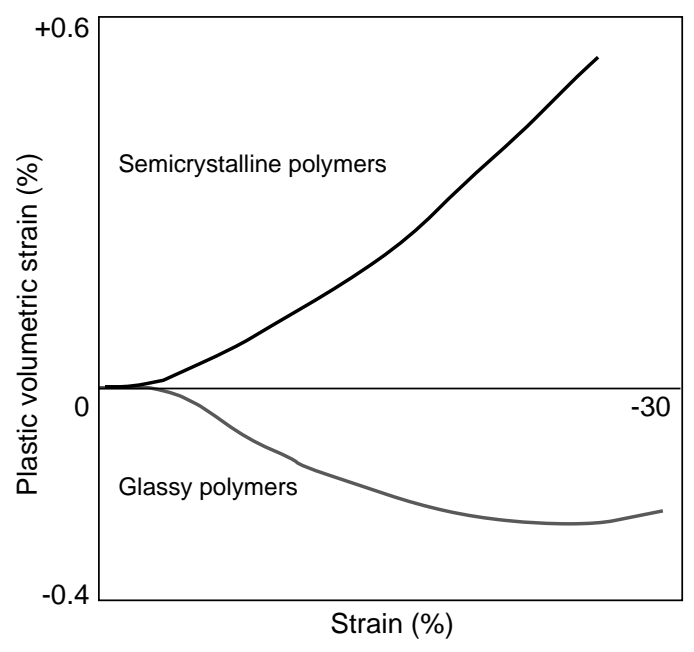

Figure 5

Schematic representation of plastic volume strain/axial strain curves in compression on various polymers.

(PP, POM and PE) undergo a net plastic expansion during the major part of the compression test. Following these tests, the authors made thin cuttings of samples for observations in a polarized light microscope. No cracks or crazes were observed. This point expresses the peculiarity of this plastic volume expansion which is certainly connected with the complexity of the microstructure of SCP and with their twophase aspect.

Since some years, numerous experimental techniques have been developed to measure the volume strain of polymers (video device, laser scan micrometer, mechanical 
extensometers, etc.). Let us note that these efforts are supported by the study of damage of pure polymers [49] or of filled polymers. These techniques are essentially applied for tensile tests which reveal mostly expansion phenomena linked to the growth of defects (cavities or crazes). In the case of uniaxial tensile tests, the volume change of a low density ethylene/butene copolymer was measured [52] and the effect of the cristallinity index on the same average and high density copolymers was studied [53]. The measures were made until large strains with a video apparatus. The results of these studies are reproduced on Figure 6 and Figure 7. For a consequent strain (about 20\%), the volume strain of the low density copolymer (PE 6) becomes widely negative. This contraction continues until $100 \%$ of axial strain. Beyond, the volume strain increases gradually and becomes positive at $200 \%$ of axial strain. This final growth of the volume seems to be connected to the appearance of a growing cavities process, associated to sample whitening in the necking zone. Physicochemical analyses (dynamic mechanical temperature analysis and raman spectroscopy) were performed to explain the initial contraction phenomenon at a molecular scale. The results indicate that one can reasonably link this contraction to a densification process inferred by the deformation of a "mesomorphic" zone, located between the crystalline lamellae and the rubberlike amorphous phase. On the other hand, when the crystallinity index increases, the contraction eases considerably and the volume strain remains positive during the last part of the test. This point indicates that the contraction effects are strictly connected to the morphology of materials and to their two-phase aspect: the contraction can become dominating only if the amorphous phase of the polymer is present in quantity. According to [52], this negative part of the volume strain is reversible after some minutes following unloading. Hence, it seems that this reversibility is associated to a recovery process, an important phenomenon at room temperature. It is not so evident to discriminate between the purely elastic effects and the irreversible aspects, because the "plasticity" of polymers is often recovered after sufficient duration or after a thermal cycle.

Let us note that for the low density polymer (PE 6), a hook of stress appears clearly (Fig. 6). The stress decreases simultaneously with the volume decrease. This observation shows that volume evolutions act directly on SCP hardening and mechanical response. These effects may be important for SCP and must, in our opinion, be integrated into the formulation of a reliable and predictive model.

The following paragraph recapitulates some results stemming from tensile tests on PA11 and $\mathrm{PVF}_{2}$, and compressive tests on PA11, performed at IFP.

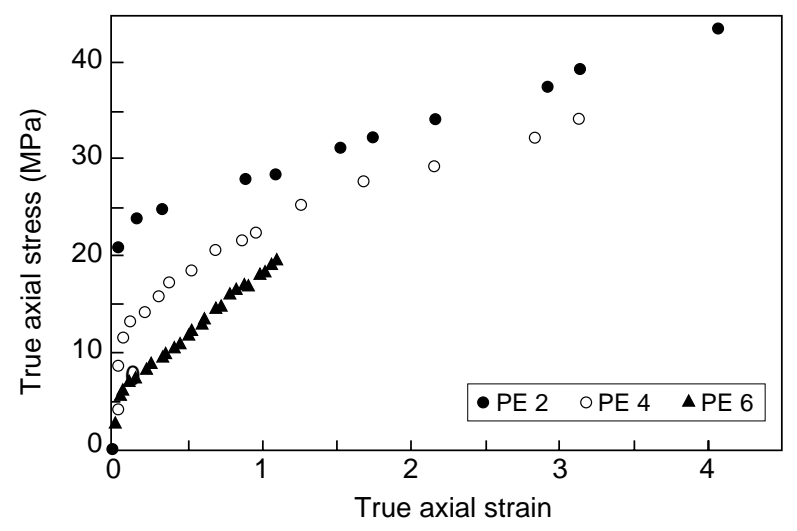

Figure 6

Axial strain/axial stress curves for high density etylene/butene copolymer: PE 2, crystallinity index $X_{c}=69 \%$; average density: PE $4, X_{c}=54 \%$ and low density: PE $6, X_{c}=32 \%$; (after [53]).

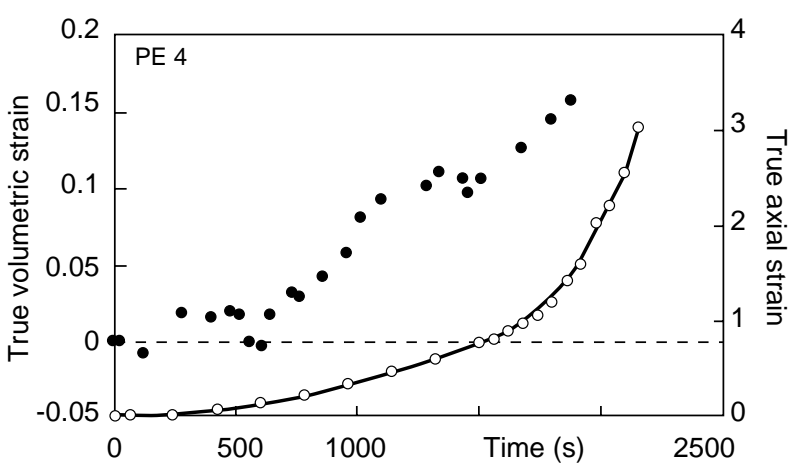

(a)

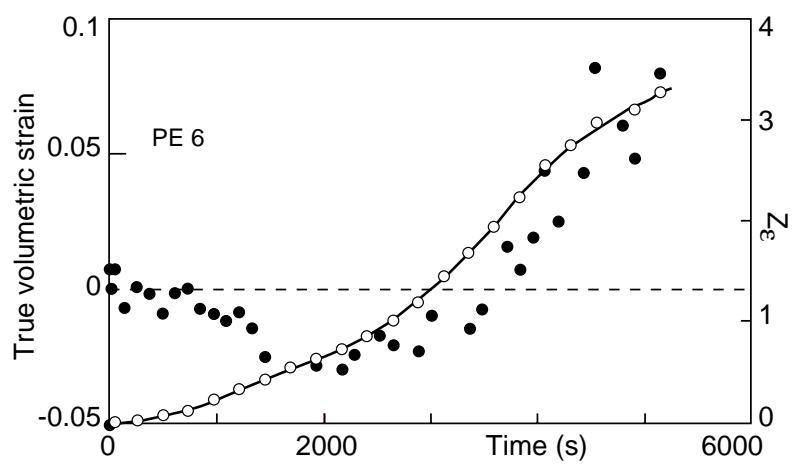

(b)

Figure 7

Axial strain/volume strain curves for average density etylene/butene copolymer. (a) PE 4, crystallinity index $X_{c}=54 \%$; (b) low density: PE 6, $X_{c}=32 \%$, (after [53]). 


\subsection{Volume Change of $\mathrm{PA} 11$ and $\mathrm{PVF}_{2}$}

\subsubsection{Materials Characteristics}

Poly(vinylidene fluoride) or $P V F_{2}$

$\mathrm{PVF}_{2}$ plates were extruded at IFP from "KYNAR 1000 $\mathrm{HD}^{\circledR}$ " granules supplied by Atochem. Their thickness is $5 \mathrm{~mm}$. The monomer is made of carbonyle and fluoride units: -[CH2-CF2]-. The average molecular weights of this polymer, obtained by gel permeation chromatography (GPC), are $M_{n} \approx 350000 \mathrm{~g} / \mathrm{mol}$ and $M_{w} \approx 600000 \mathrm{~g} / \mathrm{mol}$ and the crystallinity index, obtained by differential scanning calorimetry (DSC), is about $48 \%$. Microstructural observations by using scanning electronic microscope (SEM) showed that the studied $\mathrm{PVF}_{2}$ is constituted of small spherulites (among $2 \mathrm{~m} \mu$ and $10 \mathrm{~m} \mu$ in diameter). An analysis by small angles X-rays scattering (SAXS) allowed to estimate the long period to $150 \AA$. With a crystallinity index of $48 \%$ and supposing that the crystal is in the form of stack of lamellae, the average thickness of the lamellae is $72 \AA$ and the average interlayer distance is equal to $78 \AA$. A DMTA analysis show that the polymer presents a main glassy transition $T_{g}$ at $-40^{\circ} \mathrm{C}$ and a high transition between $50^{\circ} \mathrm{C}$ and $70^{\circ} \mathrm{C}$

\section{Polyamide 11 or PA11}

Polyamides are semicrystalline polymers with a monomer containing an amide unit separated by $\mathrm{n}$ carbonyle units: $-\left[\mathrm{NH}-\left(\mathrm{CH}_{2}\right) \mathrm{n}-\mathrm{C}=\mathrm{O}\right]-$. Their main characteristic is the presence of hydrogen bounds between two amide units of nearby chains. The average molecular weights of the polymer, obtained by GPC are $M_{n} \approx 35000 \mathrm{~g} / \mathrm{mol}$ and $M_{w} \approx 70000 \mathrm{~g} / \mathrm{mol}$. Because PA11 has a number of $\mathrm{CH}_{2}$ greater than 9 , it is necessarily semicrystalline. Crystallites gets organized in the form of lamellae within spherulites more or less well formed according to the processing conditions. The crystallinity index, determined by DSC, is $20 \%$ and the lamellae thickness is about $70 \AA$. The tests presented hereafter are performed on two PA11 which differ by the addition of coloring agents. PA11 (BESNO TL ${ }^{\circledR}$ ) was provided by Atochem. Some results of experiments performed in 1996 [54] are also introduced to illustrate the tensile volume strain of PA11.

\subsubsection{Experimental Methods at IFP}

\section{Compressive Experimental Device}

The compressive tests are performed by means of a hydraulic press developed for the study of soils and rocks. The sample, protected by an impermeable rubber coat, is placed in a triaxial cell where a confining pressure can be applied. The axial load is rate-controlled and imposed by the descent of a piston. The confining pressure is imposed through a liquid (oil) in the cell, the flow of which is controlled by a GDS pump (maximum pressure of $64 \mathrm{MPa}$ ). Samples are full PA11 cylinders injected by Atochem, $30 \mathrm{~mm}$ in diameter and $60 \mathrm{~mm}$ in height. The axial and lateral strains are measured from strain gauges stuck on the sample (Fig. 8). The confining pressure, $P_{\text {conf }}$, is applied through the fluid pressure of the cell for the lateral stress and by the descent of the piston for the axial stress. The sample is then subjected to a stress deviator by applying an axial compression.

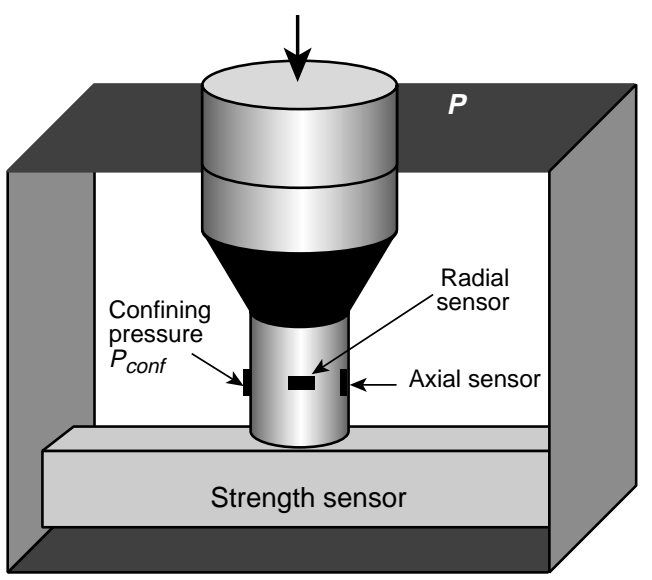

Figure 8

Geometry of the samples and position of the strain gauges.

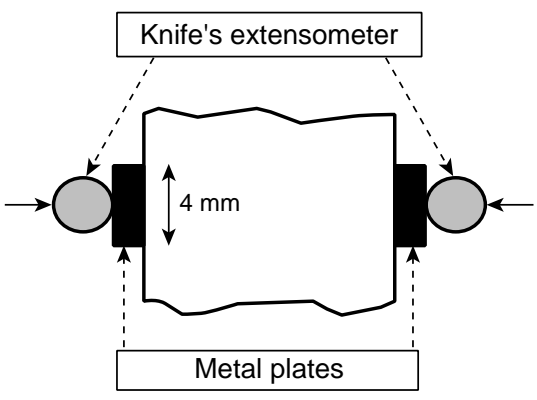

(a)

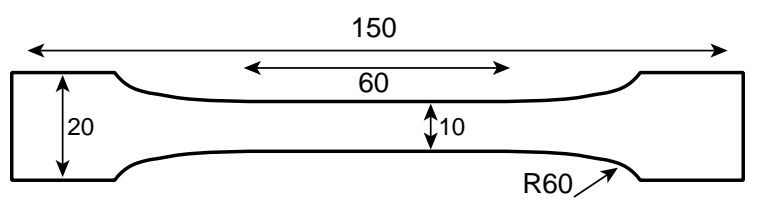

(b)

Figure 9

(a) lateral extensometer device; (b) geometry of the ISO 527 samples. 


\section{Tensile Experimental Device}

The tensile tests are performed by using an Inströn mechanical press (maximum load of $250 \mathrm{kN}$ ). The axial strain is measured with a mechanical extensometer of initial length $25 \mathrm{~mm}$. The measure of the lateral strain uses an assembly realized at IFP, from a lateral mechanical extensometer. To minimize the sinking of the lateral extensometer knives in the polymer, metal sheets are inserted between the sample and the knives, in order to better distribute the local pressure applied by the knives on the material (Fig. 9a). Dumbbell specimens ISO 527 are either machined at IFP from extruded plates, or are injected (Fig. 9b).

\subsubsection{Results on PA11}

Figure 10 and Figure 11 compare tensile tests and compressive tests at confining pressures varying from $0 \mathrm{MPa}$ to 20 $\mathrm{MPa}$, obtained at IFP on the "yellow" PA11. On Figure 10, the deviator stress $q$ is drawn versus the axial deformation. This figure shows that the effect of the mean stress is not as important as on other polymers but exists nevertheless. It seems that the behavior is identical in tension or in simple compression. This result is in agreement with those already obtained by [54] with tensile, torsion or compression tests on PA11 hollow tubes. On the other hand, as the confining pressure increases in compressive tests, the growth of the mean stress in the material induces an increase of the plastic yield stress. An interesting point concerns the convergence of the hardening of the material, for all the loading cases, up to an unique value of the axial stress of $46 \mathrm{MPa}$. This aspect seems to be in connection with the evolution of the volume strain (Fig. 11).
First of all, we obtain comparable results to those described in [51] for other SCP in compression tests. Indeed, during the first "preplastic" stage, the material tends to contract considerably. From 3\% of axial strain, the tendency is reversed and an important volume expansion appears. Let us note that the used techniques for measuring the volume change are different in both cases. This volume expansion of $\mathrm{SCP}$ in compression seems qualitatively comparable to the responses of soils and rocks. This point authorizes a comparison with geomechanics, even though SCP present some features which are not still understood. For example, the volume expansion measured on PA11 during compression increases with the confining pressure, and this fact cannot be explained by using the classic soil mechanics theory.

During the tensile tests, the volume strain globally increases. This volume expansion is associated to the elasticity of the material. On the other hand, as the material enters in the plastic domain (near the hook of stress), the total volume strain decreases lightly and increases again at the end of the test. Qualitatively identical results (Fig. 12) were obtained by [54] on PA11 (BESNO TL ${ }^{\circledR}$ ) where a contraction tendency of the volume associated to the material plastic flow in tension was measured (however, the final phase of expansion is less pronounced).

These effects, obtained in tension and compression, cannot find their origin in the Hooke's elasticity and correspond to an irreversible contracting or dilating part of the volume strain. When the axial strain increases, the volume strain declines for all the loading cases (Fig. 11). It converges to a value between $0,25 \%$ and $0,5 \%$, from $14 \%$ of axial strain (beyond 14\% of axial strain, the results must be

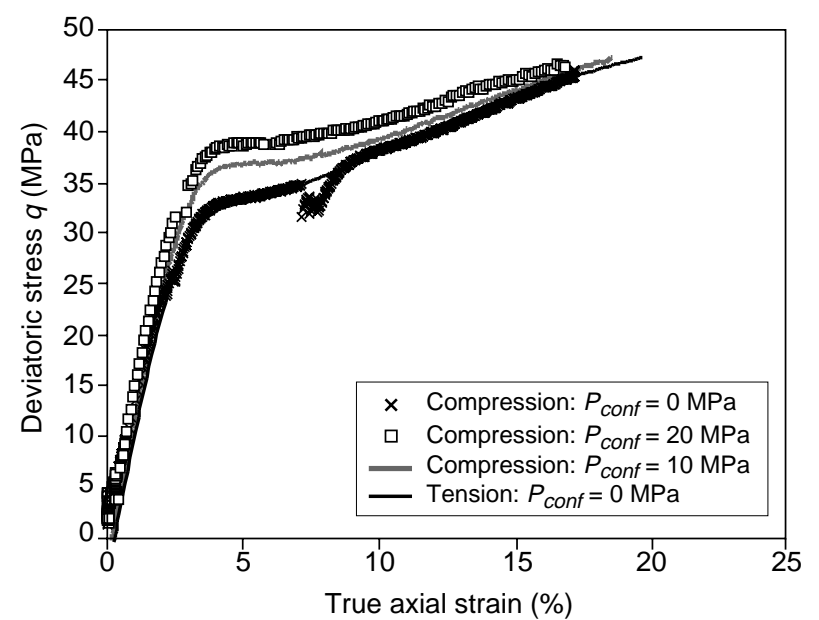

Figure 10

PA11. Deviator stress/strain curves obtained from tensile test and compressive tests at a confining pressure of 0,10 and $20 \mathrm{MPa}$.

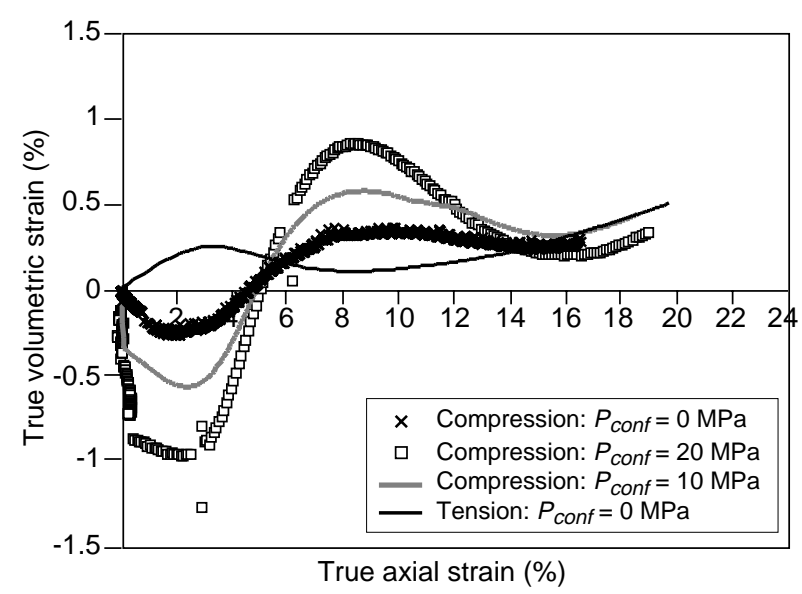

Figure 11

PA11. Axial strain/volume strain curves obtained from tensile test and compressive tests at a confining pressure of 0,10 and $20 \mathrm{MPa}$. 


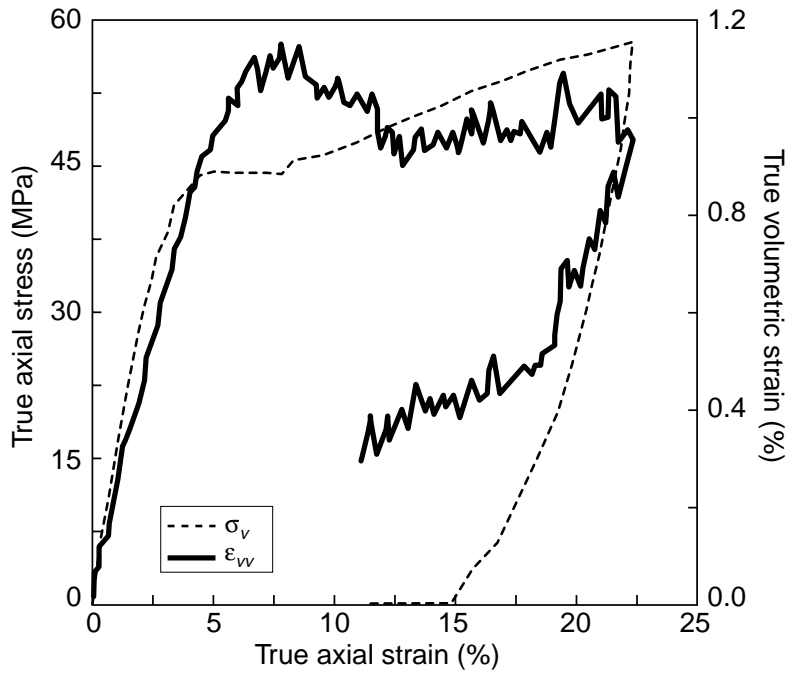

Figure 12

PA11. True stress/true strain curves and axial strain/volume strain curves obtained form tensile tests (after [54]) .

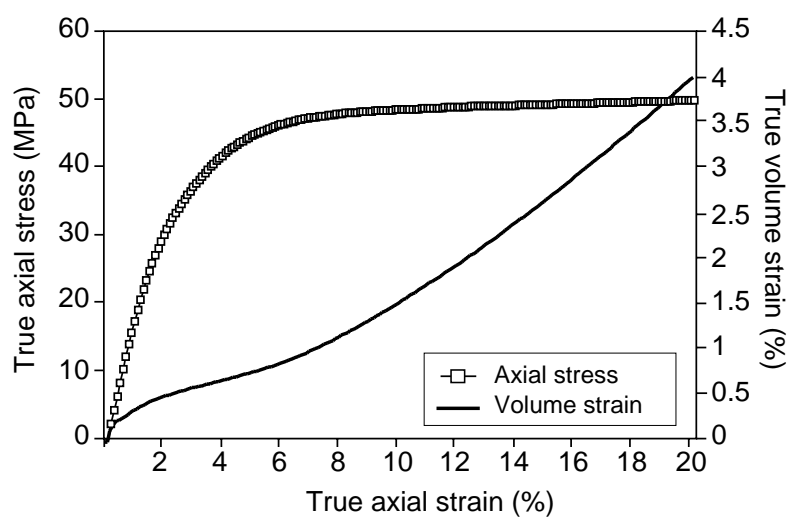

Figure 13

$\mathrm{PVF}_{2}$. True stress/true strain curves and axial strain/volume strain curves obtained from tensile tests at $23^{\circ} \mathrm{C}$.

taken with caution because the limit of the strain gauges is reached). This convergence of the volume strain seems remarkably connected to the convergence of the yield stress shown on Figure 10. On the other hand, this convergence also corresponds to a stabilization of both variables towards a constant or a critical state of $[0,25 \%, 0,5 \%]$ for the volume strain and of [46 MPa, $47 \mathrm{MPa}$ ] for the axial stress. This remarkable point of the behavior will be considered hereafter in the mechanical model (Section 4).

\subsubsection{Results on $\mathrm{PVF}_{2}$}

Only tensile tests were performed on $\mathrm{PVF}_{2}$. The measure of the volume strain was approached by several laboratories, with different experimental devices. At room temperature, in spite of the inevitable scattering, the obtained results match and indicate a strong volume expansion, starting from $5 \%$ of axial strain [49] and [55]. Figure 13 shows results obtained at IFP on samples machined from extruded plates.

The mechanical behavior differs from that of PA11. As regards the stress, the hook of stress is replaced by a regular "knee". As regards the volume strain, a very weak contraction is obtained at the beginning of the test, followed by a strong expansion which reaches about $4 \%$ at $18 \%$ of axial strain. This result is confirmed by [56] whereas, in the experiments performed by [55], the laser scan micrometer measures give a more important volume expansion (about $10 \%$ for the same axial strain). One can explain this difference by different extrusion processing of $\mathrm{PVF}_{2}$.

The influence of the temperature on the $\mathrm{PVF}_{2}$ volume strain evolution has been studied recently [56, 57]. The obtained results are out of the scope of the present paper. Nevertheless, we show below an important result, concerning the volume strain evolution during a creeping period of $11000 \mathrm{~s}$ at $80^{\circ} \mathrm{C}$ [57]. At this temperature, for homogeneous tensile test, the volume strain globally increases when the test is not interrupted (Fig. 14a). Here, the elastic effects are dominant and the plastic phenomena do not demonstrate themselves explicitly. However, for creeping test, when the tensile test is interrupted and the axial stress is kept constant, the material stores no more elastic energy and the obtained volume strain represents the plastic volume behavior of the material. A significant contraction of the polymer during all the creeping test is obtained (Fig.14b). Let us note that, at this temperature, the amorphous phase is completely in a rubberlike state (free amorphous) and represents more half of the material. Conversely, this phenomenon did not appear at room temperature for which the quantity of the free amorphous is not important. In this case, a significant plastic expansion indicates the presence of a prevailing cavities growth process [55] and [57].

\subsubsection{Synthesis}

All these results shows that several physical processes are at the origin of the volume change of SCP. From a macroscopic and phenomenological point of view, these processes make appear effects of contraction during the first stages of the deformation, followed by a more or less important expansion depending on the SCP nature. This expansion is not necessarily produced by the same physical phenomena. For example, PA11 expansion is relatively weak and does not seem connected to a cavity growth or to a damaging process. Conversely, in the case of $\mathrm{PVF}_{2}$, the whitening of specimens at room temperature indicates the growth of defects [49]. The physical origin of the plastic contraction, is not still well clarified. It is certainly linked to the two-phase aspect of the SCP and to their microstructural complexity. So, these two phenomena: plastic contraction and dilation seem to coexist. One of them becomes dominant when certain conditions of loading rate, deformation and temperature are gathered. 

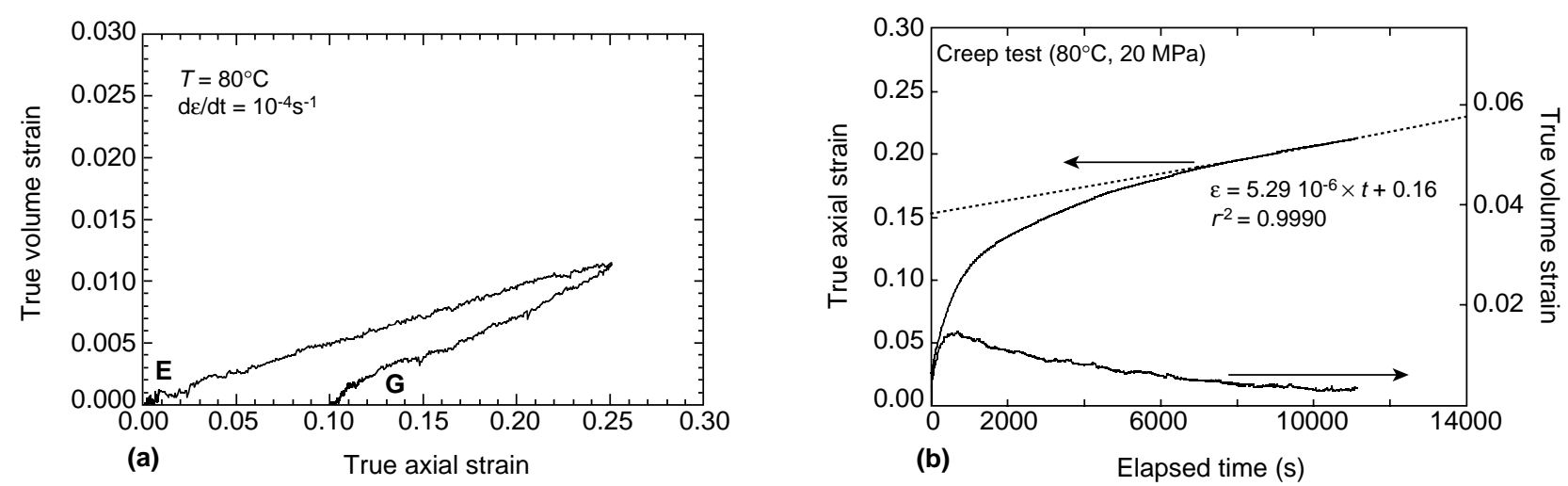

Figure 14

$\mathrm{PVF}_{2}$. (a) volume strain/true axial strain curve for homogeneous tensile test at $80^{\circ} \mathrm{C}$; (b) creeping time/true axial strain curve and creeping time/volume strain curve obtained from a creep tensile test at $80^{\circ} \mathrm{C}$ (after [57]). .

\section{BASIS OF THE TWO-PHASE APPROACH}

\subsection{Microstructure of SCP}

This section recalls some essential elements of the structural morphology of SCP. A more detailed description can be found in [58]. In the melted state, the macromolecules are in an isotropic disordered state which corresponds to the amorphous state. When the melted material is cooled, two mechanisms may appear: the germination and the crystalline growth. The crystallinity results from regular stacks of macromolecular chains. Because of chain ends, the order cannot be total in a polymer: hence, it is semicrystalline [59]. The morphology of industrial thermoplastic polymers is a two-phase mixture of amorphous zones and crystalline zones deeply intricate. The microstructure generally consists of spherulites, that is of radially aggregated crystalline lamellae around a nucleation germ. These lamellae are separated and connected by amorphous macromolecules. The spherulitic structure can be revealed by using various means. For example, by using the optical polarized light microscope, spherulites appear in the form of Maltese cross. By making an adequate chemical attack, it is possible to dissolve the amorphous part surrounding spherulites. Then, their structure can be observed in three dimensions by using a SEM analysis (Fig. 15a). Some images also allow to realize the complexity of the crystalline network which gets organized in the form of trees from nucleation germs (Fig. 15b). So, the SCP morphology involves several scales of observation. At the molecular scale, the basic motive imposes the elementary physicochemical features of the material. The crystalline network is organized through different scales which involve more and more complex and elaborated physical entities: crystalline lamellae, spherulites and set of spherulites (Fig. 16). The parameters influencing the crystallinity are mainly the

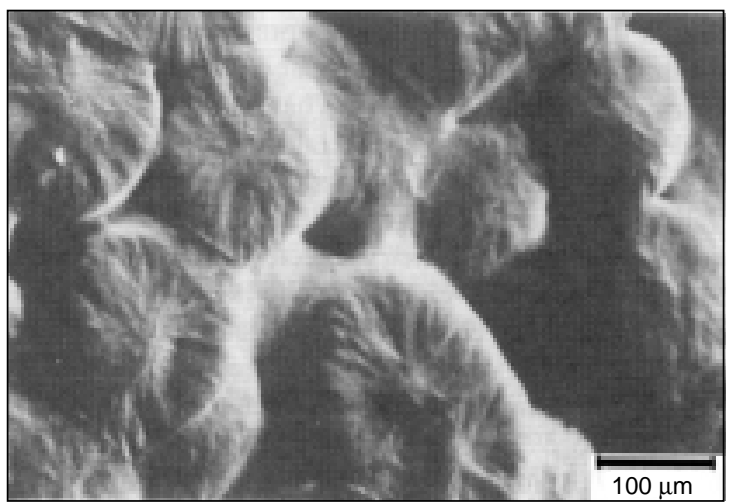

(a)

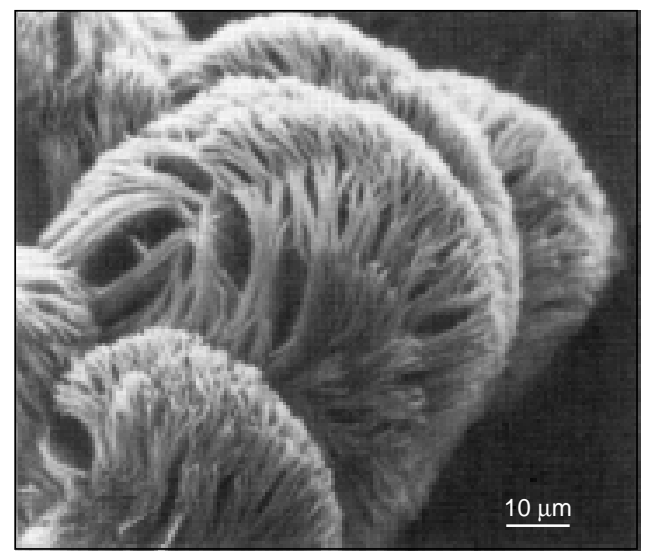

(b)

Figure 15

SEM microphotographs of melt crystallized spherulites formed under unrestricted growth conditions. (a) trans-1, 4-poly isoprene fraction of $M_{v}=1.4 \times 10^{5}$ (crystallized from amlyacetate $(1 \%$ solution) at $20^{\circ} \mathrm{C}$ and reacted with $\mathrm{OsO}_{4}$.

(b) SEM microphotograph of PBFP cast from solution of THF at room temperature After [60].

Reprinted from "Journal of Materials Science", with permission from Klurver Academic. 


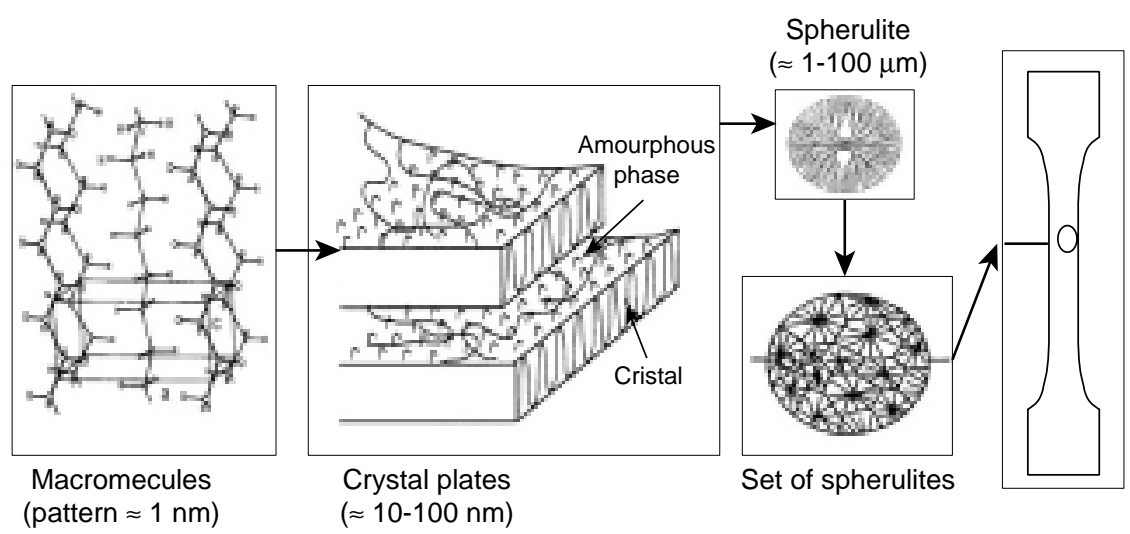

Figure 16

Multi-scale representation of the microstructural morphology of SCP.

molecular structure, the molecular weight, the presence of plasticizers and especially the processing (thermal-mechanical history). The processing (molding, injection, extrusion) determines the material morphology: for example, the spherulites shape (spherical, flattened ellipsoid, discs in shape of sheaves) is influenced by the flow intensity [58].

\subsection{Two-Phase Interpretation of the Microstructure}

With regard to the intrinsic behavior of the material, a phenomenological model will be really interesting only if it leans on multi-scale analyses: from the microscopic scale, where the deformation of macromolecules is heterogeneous, to the macroscopic scale, where the deformation is homogenized. Most of the approaches made so far consider that, above the glassy transition, the amorphous phase is homogeneous with a rubberlike behavior. In fact, physicochemical observations show that the amorphous part of the polymer has an heterogeneous structure due to the presence of crystallites which reduce locally the molecular mobility. This notion applied to SCP $[61,62]$ is inspired by a model initially developed for filled rubbers [63]. For these materials, it was shown that black carbon particles restrict the mobility of certain parts of the rubberlike amorphous [64]. The notion of "extended glassy transition" is developed in [61, 62]: there is a range of temperature in which a part of the amorphous phase is in a rubber state, a part is in a glassy state and the other parts have just crossed their glassy transition $\left(T_{g}\right)$. A first approximation consists in admitting a partition of the amorphous in "tied or confined amorphous" whose mobility is reduced and in "free amorphous" corresponding to the part of chains behaving as the amorphous in a rubberlike state (Fig. 17).

The main consequence of the reduction of the amorphous mobility is that the glassy transition is spread towards higher

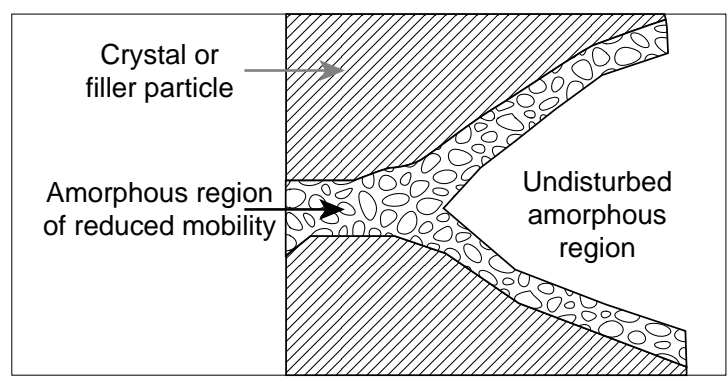

Figure 17

Amorphous partition in confined (or tied amorphous) and in free amorphous, for filled rubbers or SCP (after [61]).

temperatures. This phenomenon is put in evidence through DMTA testing where the loss peak $(\tan \delta)$ is pushed away towards the "high" temperatures (Fig. 18). The validity of this model is confirmed by the study of the physical aging of several materials $[61,62]$. Below $T_{g}$, the amorphous phase is completely glassy and aged in the same way as a pure amorphous material. Above $T_{g}$, a part of the amorphous of SCP is still glassy and the effects of aging continue to demonstrate themselves. Then, a fine analysis of different cases of aging allows to define transition domains, during which both parts of the amorphous cross successively their glassy transition. This leads to define a low transition temperature $T_{g}^{L}$, under which both parts of the amorphous are in a glassy state, and a high transition temperature $T_{g}^{U}$, above which both parts are in a rubberlike state. Figure $19 \mathrm{~b}$ shows the simplistic evolution of the shift rate factor $\mu$, deduced from creep curves obtained for various times of aging 
(Fig. 19a). The high and low transition temperatures can be explicitly determined from this diagram. For PA6, it is possible to define a low transition at $-110^{\circ} \mathrm{C}$ and a high transition at more than $100^{\circ} \mathrm{C}$, while the main glassy transition is around $40^{\circ} \mathrm{C}$.

This partition of the amorphous phase in free amorphous and tied amorphous has been justified, for PA11, by another experimental technique. To study the links between the macroscopic mechanical deformation and the structural evolution, an infrared spectroscopy study (FT-NIR) was performed, in association with the Essen University in Germany [65]. An aspect of this study concerned results obtained by deuteration (replacement of hydrogen $\left(\mathrm{H}_{2}\right)$ by deuterium $\left(\mathrm{D}_{2}\right)$ in the macromolecules) and by rheo-optical measures on the PA11 saturated by various products. PA11 films were immersed in $5 \mathrm{ml}$ of several deuteration agents $\left(\mathrm{D}_{2} \mathrm{O}\right.$, d-methanol, d-ethanol, d-propanol, d-1-butanol , d-1pentanol, d-t-butanol, d-t-pentanol) at $50^{\circ} \mathrm{C}$. Spectra were measured regularly to follow the progress of the exchanges NH/ND. The percentage of accessibility (Z\%) of the deuteration agent was estimated by:

$$
Z(\%)=\left[1-\frac{A v(X H)}{A v(X H)_{i}}\right] 100
$$

where $A$ is the absorbance and the subscript $i$ refers to the intensity of the selected absorption band $X H$, of the nondeuterated sample. On Figure 20, a diagram drawing the accessibility of the amorphous according to the deuteration time is given for the whole tested agents. Whatever was the molecule size of the deuteration agent, only $50 \%$ (at most) of total amorphous was accessible, even after $75 \mathrm{~h}$ of deuteration. Note that the experiments were performed $10^{\circ} \mathrm{C}$ above the main $T_{g}$.

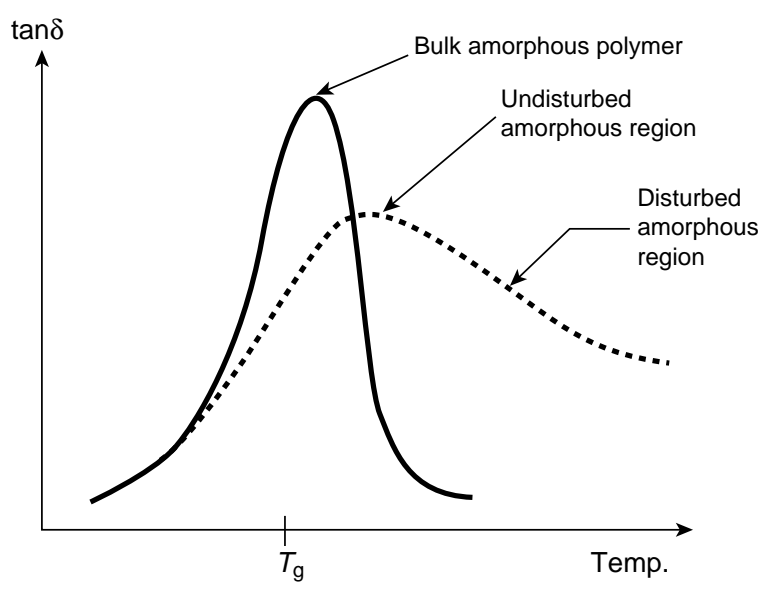

Figure 18

DMTA analysis and extended glassy transition for SCP and filled rubbers (after [61]).
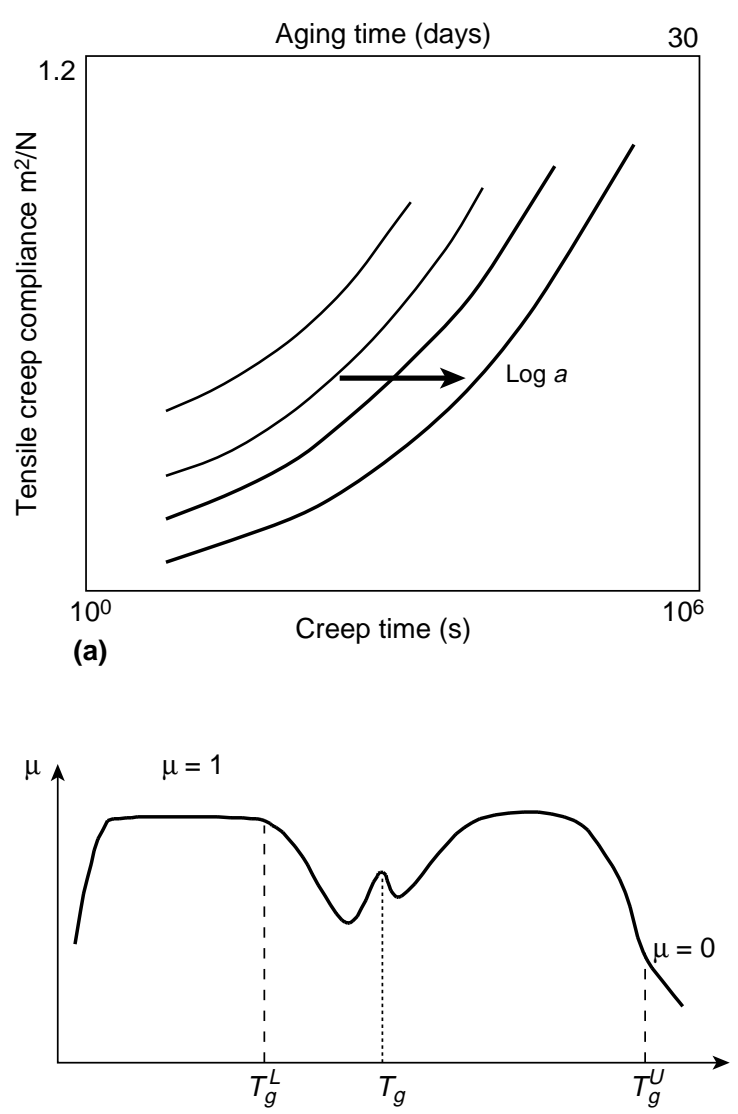

(b)

Figure 19

(a) Schematic creep curves for many aging time of PSC; (b) schematic representation of the shift rate factor of creeping curves, defined by $\mu=d \log a / d \log t_{e}$ allowing the definition of the low and high transitions.

It is generally admitted that diffusion of gases in SCP takes place in the amorphous phase [66]. The obtained results seem emphasize that the mobility of half the amorphous is considerably reduced and opposes to the diffusion of isotopes. This part of the amorphous is then comparable to a confined or tied amorphous zone. So, the diffusion takes place essentially in the free amorphous, outside the crystalline and tied amorphous zones (at least for the tested molecules). In a schematic way, one can so consider that the free part of the amorphous represent an exchange zone of the material with its outside environment. In real and industrial situation, this zone consists of macromolecules but also of various other molecules: plasticizer, additives, molecules of gases, molecules of water, etc.

The physical considerations presented above allow us to propose a pragmatic modeling approach, by admitting at once a representation of the microstructure, by two homogeneous phases in deep interaction at a mesoscopic scale. 


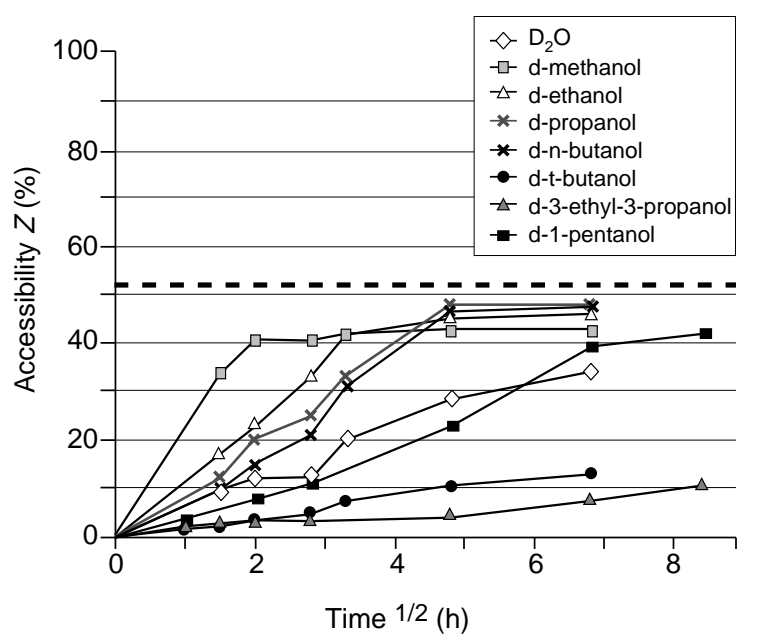

Figure 20

Rheo-optical FT-NIR-spectroscopy of polyamide 11 (deuterated): accessibility/time ${ }^{1 / 2}$ diagram at $50^{\circ} \mathrm{C}$.

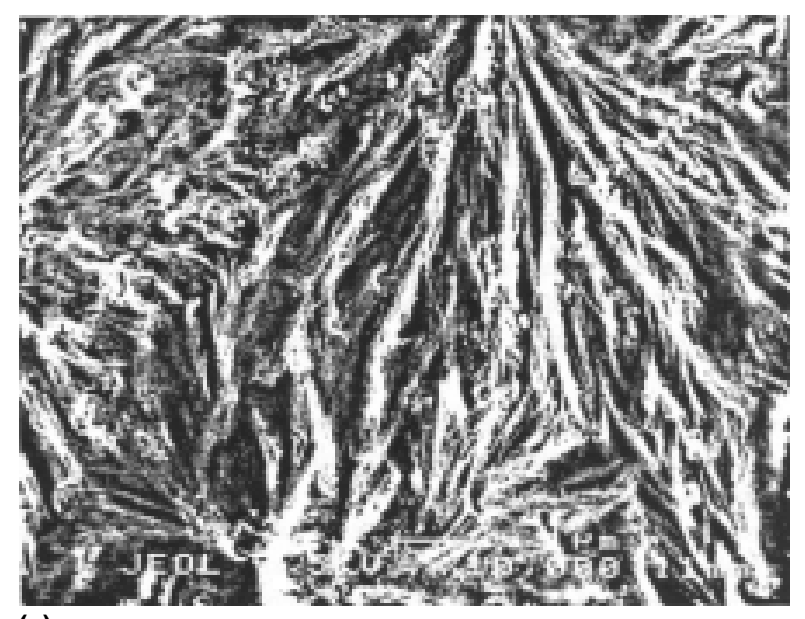

(a)

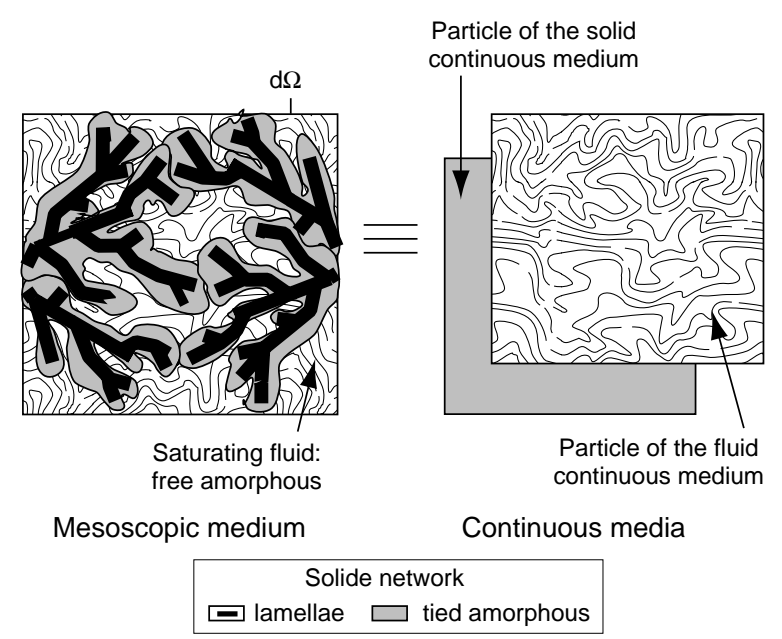

Figure 21

Two-phase representation of the several parts of the microstructure at a mesoscopic scale and scaling to the continuum.

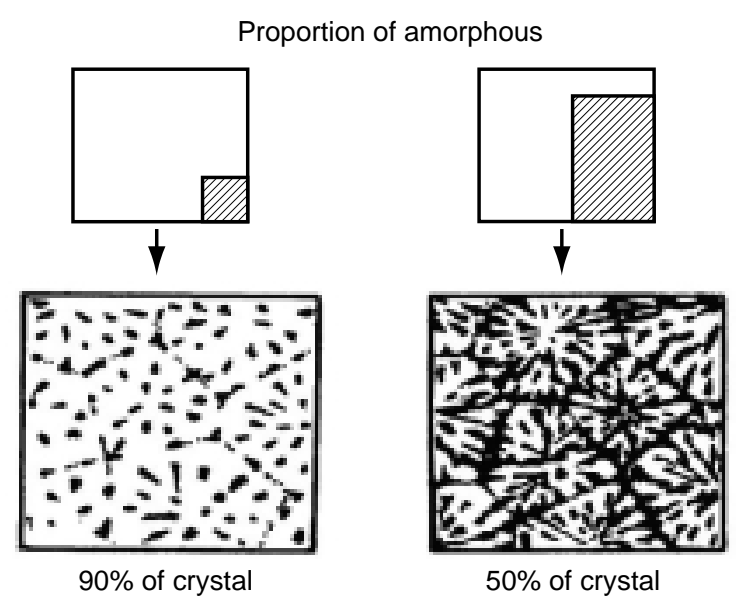

(b)

Figure 22

Interconnected space and localization of the free amorphous (a) SEM microphotography of slowly crystallized PVF $_{2}$ showing the boundary between 3 spherulites (after [67]) and (b) schematic representation of the distribution of the interspherulitic amorphous (after [68]).

The distinction between the various components of the polymer (crystalline zones, confined amorphous and free amorphous) can be effected on the basis of mechanical and rheological hypotheses. For the sake of simplicity, only two phases of the material are considered according to their mechanical characteristics. The first phase consists of crystalline lamellae surrounded with amorphous in a confined or "glassy" state. Here, the stiffness of constituents is high and the behavior of this phase is that of a "solid". The second consists of free amorphous, but also of a certain quantity of plasticizers and molecules of gas resulting from possible diffusion phenomena during the life of the material.
This set constitutes a mixture in the sense of thermodynamics and its behavior is supposed to be that of a "fluid" (Fig. 21). Indeed, the free amorphous phase has a relatively weak stiffness with regard to that of the other parts. This "soft" phase is characterized by a raised enough intrinsic mobility. In first estimate, shearing in this phase is supposed to be negligible with regard to the rest of the solid, at least in the case of the small and average strains.

The set (crystalline lamellae + tied amorphous) constitutes, at the mesoscopic scale, a complex solid network organized around the crystalline growth. A means to reveal this particular topology is to make a chemical attack to suppress 
the amorphous part of the material. The freed space, initially occupied by the amorphous is then comparable to an interconnected pseudo porosity. Free amorphous is so comparable to a fluid which saturates the complementary space to that of the solid structure. When the proportion of amorphous increases, the crystalline density decreases and the pseudo porosity (free connected space) increases (Fig. 22).

\subsection{Thermodynamic Framework}

The previous representation of the microstructure allows us to consider that a semicrystalline polymer immersed in a natural environment is comparable to an open system $[38,42$, 69]. Indeed, a certain quantity of products is susceptible to cross it at any time, with a kinetics of diffusion appropriate for the various species of the system. The complete and rigorous description of these physical processes is out of the scope of the present paper. However, considering these aspects of SCP behavior, the framework of the thermodynamics of open systems is used to derive an explicit coupling equation between the "solid" part and the "fluid" or "soft" part of the material. In this first work, we consider that plasticizers and diffusive products occupy the same pseudo porous space as the free amorphous. This space is characterized by the proportion of free amorphous $\phi_{a}$. This quantity is directly linked to the global proportion of amorphous $\Phi_{a} . \phi_{a}$ evolves according to the temperature and follows the various transitions of the material. A difficulty of this approach is to quantify exactly the value of $\phi_{a} . \phi_{a}$ depends obviously on the crystallinity index (CI): high values of CI correspond to low values of $\phi_{a}$. When the temperature is over the high transition temperature (if it exists), $\phi_{a}=\Phi_{a}$. For PA11, the results of deuteration (Fig. 20) can also be used and, if the temperature is around $T_{g}, \phi_{a}$ represents $50 \%$ of the total amorphous phase.

In the following, the thermodynamic basis of the model is presented. The basic postulate consists in proposing a heuristic scale transition, supposing that the elementary representative volume (ERV) in the mesoscopic scale can be suitably represented at the macroscopic scale, by superimposing two continua in deep interaction (Fig. 21). Here, the exact geometry of the microscopic medium is not described explicitly. This method allows to overcome the complexity inherent to a homogenization approach which would be applied to the complicated topology of the microstructure. It is made possible by a partition of the medium in a hard phase and a soft phase, the composition of which can vary with temperature.

Let us consider a solid body occupying a material volume $\Omega$, bounded by a surface $\Gamma$. The medium, being an open system, a quantity of mass $m$ can get through $\Gamma$. This mass contribution $m$ corresponds to the exchange of the fluid mixture with the exterior, constituted of amorphous, gas or water molecules and plasticizers. The approach developed for the porous continuum at the mesoscopic scale consists in privileging the movement of the solid skeleton with regard to that of the fluid. The equations of conservation are written according to this basic postulate. Let $\underline{x}$ be the position of a particle of the solid skeleton in the deformed configuration, and $\underline{X}$ the corresponding position in the reference configuration. For every constituent $i$ of the fluid, a relative kinematical rate of the fluid with regard to the skeleton is introduced:

$$
\underline{v}_{i}^{r}(\underline{x}, t)=\underline{v}_{i}^{f}(\underline{x}, t)-\underline{v}^{s}(\underline{x}, t)
$$

where $\underline{v}_{i}^{f}(\underline{x}, t)$ and $\underline{v}^{s}(\underline{x}, t)$ are respectively absolute rates of the fluid constituent and the absolute rate of the skeleton. Contrary to the general case of the unsaturated porous media, the various constituents are not in distinct phases in the polymer. They occupy the same space comparable to an unique medium. The fluid phase is equivalent to a molecular mixture. The relative molar rate of the mixture with regard to the skeleton is defined as:

$$
\underline{v}_{m}^{r}(\underline{x}, t)=\frac{\sum_{i} n_{i} \underline{v}_{i}^{r}(\underline{x}, t)}{\sum_{i} n_{i}}
$$

where $n_{i}$ is the number of moles of the $i^{\text {th }}$ constituent. Then the eulerian mass flux of fluid mixture is written as:

$$
\underline{w}_{m}(\underline{x}, t)=\rho_{m} \phi_{a} \underline{v}_{m}^{r}(\underline{x}, t)
$$

where $\rho_{m}$ is the fluid mixture density. Transport formulae allow to define a Lagrangian flux by:

$$
\underline{M}_{m}(\underline{X}, t)=J(\underline{X}, t)[\underline{\underline{F}}(\underline{X}, t)]^{-1} \cdot \underline{w}_{m}(\underline{x}, t)
$$

where:

$$
\underline{\underline{F}}(\underline{X}, t)=\underline{\underline{\operatorname{Grad}}}(\underline{x}, t) \quad \text { et } \quad J(\underline{X}, t)=\operatorname{det} \underline{\underline{F}}(\underline{X}, t)
$$

Then, the volume density of internal energy of the system $E$ and its volume entropy density $S$ are defined by:

$$
\begin{aligned}
& E d \Omega=E_{s} d \Omega+E_{m} d \Omega \\
& S d \Omega=S_{s} d \Omega+S_{m} d \Omega
\end{aligned}
$$

where $E_{s}$ is the volume density of internal energy of the solid skeleton, $S_{s}$ is the entropy of the solid skeleton, while $E_{m}$ is the volume density of internal energy of the fluid mixture and $S_{m}$ is the entropy of the fluid mixture. The following fluid mixture thermodynamic quantities are also defined:

$$
H_{m}=E_{m}+\frac{P_{m}}{\rho_{m}}: \text { fluid mixture enthalpy. }
$$


$G_{m}=E_{m}+\frac{P_{m}}{\rho_{m}}-T S_{m}$ : free enthalpy of the fluid mixture

where $T$ is the absolute temperature.

The free enthalpy of the fluid mixture is connected to the constituents of the mixture by the following molar relation:

$$
G_{m}=\sum \mu_{i} n_{i}
$$

where $\mu_{i}$ is the chemical potential of the $i^{\text {th }}$ constituent and $n_{i}$ its number of moles. The equations of energy conservation, mass conservation, the entropy inequality and the application of the Green's theorem lead to the local Clausius-Duhem's inequality:

$$
\begin{aligned}
\mathrm{D}=\underline{\underline{\pi}}: \underline{\dot{\Delta}}+G_{m} \dot{m}-S \dot{T}-\dot{\Psi}+G_{m} \dot{m}-\underline{\underline{M}}_{m} \underline{\operatorname{grad}} G_{m} \\
-\frac{\underline{\underline{Q}}}{T} \underline{\operatorname{grad} T} \geq 0
\end{aligned}
$$

where derivation of a quantity $y$ with time is denoted $\dot{y}=\frac{\mathrm{d} y}{\mathrm{~d} t}$.

In the Expression (13), $\underline{\underline{\pi}}$ is the stress tensor of PiolaKirchhoff, $\triangleq$, the strain tensor of Green-Lagrange, $\Psi$, the volume density of free energy of the whole system and $Q$, the heat flux. For the sake of simplicity, we adopt afterward the hypothesis of small perturbations for the extensive variables. Under this hypothesis, one can suppose an additive decomposition of the strain and of the fluid mixture mass contribution into an elastic part and a viscoplastic part:

$$
\begin{aligned}
& \underline{\underline{\varepsilon}}=\underline{\underline{\varepsilon}}_{v p}+\underline{\underline{\varepsilon}}_{e} \\
& m=m_{v p}+m_{e}
\end{aligned}
$$

where $\underline{\underline{\varepsilon}}_{v p}$ and $\underline{\underline{\varepsilon}}_{e}$ are the viscoplastic and linearized elastic strain tensors.

Then, a "plastic connected pseudo porosity" occupied by the fluid mixture is introduced by:

$$
\phi_{v p}=\frac{m_{v p}}{\rho_{0}^{m}}
$$

where $\rho_{0}^{m}$ is the density of fluid mixture in the reference state.

The classic hypothesis of uncoupling the dissipations and (13) lead to:

$$
\begin{gathered}
\mathrm{D}_{1}=\underline{\underline{\sigma}}: \underline{\underline{\dot{\varepsilon}}}+G_{m} \dot{m}-S \dot{T}-\dot{\Psi} \geq 0 \\
\mathrm{D}_{2}=-\underline{M}_{m} \underline{\operatorname{grad}} G_{m} \geq 0 \\
\mathrm{D}_{3}=-\frac{\underline{\underline{g}}}{T} \underline{\operatorname{grad}} T \geq 0
\end{gathered}
$$

The inequality (17) is the intrinsic dissipation. The inequality (18) is the dissipation due to the transport of the fluid mixture through the solid skeleton. This movement is defined by the notion of average molar rate (4) of all the fluid constituents. Note that, for SCP, in most of the cases, the constituent corresponding to the free amorphous macromolecules do not move with regard to the skeleton and its relative rate will be taken equal to zero. The inequality (19) is the thermal dissipation.

We assume that the evolution of the continuum is governed by a set of state variables, on which depends the free energy:

$$
\Psi=\Psi\left(\underline{\underline{\varepsilon}}_{e}, \underline{\underline{\varepsilon}}_{v p}, m, \phi_{v p}, \gamma_{i}\right)
$$

where $\gamma_{i}$ are internal state variables. Then, inequality (17) and relation (20) allow to obtain the following equations of state:

$$
S=-\frac{\partial \Psi}{\partial T} \quad \underline{\underline{\sigma}}={\frac{\partial \Psi}{\partial \underline{\underline{\varepsilon}}_{e}}} \quad G_{m}=\frac{\partial \Psi}{\partial m}
$$

By using (21), the intrinsic dissipation $\mathrm{D}_{1}$ is:

$$
\mathrm{D}_{1}=\underline{\underline{\sigma}}: \underline{\underline{\varepsilon}}_{v p}+G_{m} \dot{\phi}_{v p}+\sum_{i} A_{i} \dot{\gamma}_{i} \geq 0
$$

where the thermodynamic affinities associated to the internal state variables derive from the following equation of state:

$$
A_{i}=\frac{\partial \Psi}{\partial \gamma_{i}}
$$

Now, if the plastic porosity is defined as:

$$
\phi_{v p}=\underline{\underline{B}}_{v p}: \underline{\underline{\varepsilon}}_{v p} \text { with } \underline{\underline{B}}_{v p}=\beta \underline{\underline{1}}
$$

the dissipation (21) becomes, thanks to (24):

$$
\mathrm{D}_{1}=\left(\underline{\underline{\sigma}}+\beta G_{m} \underline{\underline{1}}\right): \underline{\underline{\varepsilon}}_{v p}+\sum_{i} A_{i} \dot{\gamma}_{i} \geq 0
$$

This inequality shows that the plastic dissipation is associated to the following effective stress tensor:

$$
\underline{\underline{\sigma}}_{\beta}^{\prime}=\underline{\underline{\sigma}}+\beta G_{m} \underline{1}
$$

The stress tensor (26) can be decomposed into a deviatoric part and a spherical part:

$$
\underline{\underline{\sigma}}_{\beta}^{\prime}=\underline{\underline{\operatorname{dev}}}\left(\underline{\underline{\sigma}}_{\beta}^{\prime}\right)+\frac{1}{3} \operatorname{trace}\left(\underline{\underline{\sigma}}_{\beta}^{\prime}\right)=\underline{\underline{S}}_{\beta}+P_{\beta} \underline{\underline{1}}
$$

The inequalities (18), (19) and (25) should be verified for all real evolutions of the system. This condition is verified if the 
thermodynamic affinities associated to the dissipations derive from a convex potential or from a convex pseudo-potential:

$$
\begin{gathered}
\left(\underline{\underline{\sigma}}_{\beta}^{\prime}, A_{i}\right) \in \partial \Phi_{1}\left(\underline{\underline{\varepsilon}}_{v p}, \dot{\gamma}_{i} ; \underline{\underline{S}}_{\beta} ; P_{\beta}\right) \\
-\underline{\operatorname{grad}} G_{m} \in \partial \Phi_{2}\left(\underline{M}_{m}\right) \\
-\underline{\operatorname{grad}} T \in \partial \Phi_{3}\left(\frac{\underline{Q}}{T}\right)
\end{gathered}
$$

These equations define a rigorous thermodynamic framework, from which constitutive equations of the continuum may be developed without violation of both laws of thermodynamics. So, the equations of state (21) and (23) and the complementary laws (28)-(30) are physically based equations which define completely the thermal, mechanical and diffusive behavior of SCP.

\section{CHOICE OF THE FREE ENERGY POTENTIAL}

Here, we only consider isothermal evolutions and monotonic loading. To describe the reversible part of the behavior, we make the hypothesis of small perturbations for the elastic strain and for the fluid mass contribution. This constitutes a physical linearization of the behavior where the elastic part is supposed to be linear. By making a second order Taylor's expansion of $\Psi$, the following quadratic expression for the free energy is written for an isotropic medium:

$$
\begin{aligned}
& \Psi=\underline{\underline{\sigma}}^{0}: \underline{\underline{\varepsilon}}_{e}+m \Psi_{m}^{0}+P^{0}\left(m / \rho_{m}^{0}-\phi_{v p}\right)+\frac{1}{2} \underline{\underline{\varepsilon}}_{e}: \underline{\underline{\underline{C}}}: \underline{\underline{\varepsilon}_{e}} \\
& -\left(m / \rho_{m}^{0}-\phi_{v p}\right) M b \varepsilon_{e}^{v o l}+\frac{1}{2} M\left(m / \rho_{m}^{0}-\phi_{v p}\right)^{2}+U\left(\gamma_{i}\right)
\end{aligned}
$$

in which:

$\gamma_{i} \quad$ is a set of internal state variables associated to the plastic hardening of the material.

$U(\gamma) \quad$ is the energy stored in the material during the irreversible evolutions.

$\underline{\underline{\sigma}}^{0} \quad$ is the initial internal stress tensor.

$\Psi_{m}^{0}, G_{m}^{0}, \rho_{m}^{0}$ and $P^{0}$, are the free energy of the fluid mixture, the free enthalpy, the mixture density and its pressure in the reference state, with $\Psi_{m}^{0}=G_{m}^{0}-P_{0} / \rho_{m}^{0}$.

$\varepsilon_{e}^{\mathrm{vol}} \quad$ is the elastic part of the volume strain.

$M$ and $b \quad$ are coupling parameters linked to the compressibility of the phases.

$\underline{\underline{\underline{C}}} \quad$ is the Hooke's tensor of elasticity for the twophase continuum (apparent elasticity).
By using (21) and (23) the constitutive equations yield:

$$
\begin{gathered}
\underline{\underline{\sigma}}=\underline{\underline{\sigma}}^{0}+\underline{\underline{\underline{\underline{C}}}}: \underline{\underline{\varepsilon}}_{e}-M b\left(\frac{m}{\rho_{m}^{0}}-\phi_{v p}\right) \\
G_{m}=G_{m}^{0}+\frac{M}{\rho_{m}^{0}}\left[-b \varepsilon_{e}^{\mathrm{vol}}+\left(\frac{m}{\rho_{m}^{0}}-\phi_{v p}\right)\right] \\
A_{i}=\frac{\partial U}{\partial \gamma_{i}}
\end{gathered}
$$

The previous system is valid only if the free enthalpy $G_{m}$ of the mixture can be linearized. In that case (33) bring in only the first order terms of $G_{m}$. The Equations (32) and (33) describe the coupling between the solid phase (crystalline lamellae and confined amorphous) and the fluid phase (free amorphous and additives). The Equation (33) contains a constitutive term which introduces the effect of an exchange of constituents with the outside environment on the twophase system. To describe more precisely the model, the particular situation, where there are no exchanges between the material and the immediate environment, is considered. In that case, the mass contribution $m$ is null and the system behaves as a closed one.

The specific choice of the fluid free enthalpy allows to completely define the constitutive equations. This is equivalent to derive an equation of state for the free amorphous, assimilated here to an amorphous above its $T_{g}$, i.e. in a liquid state. Several equations of state exist in the literature. The most sophisticated are deduced from statistical thermodynamics [70-73]. In the present work, the pressure is supposed to be in the range of $0,1 \mathrm{MPa}(1 \mathrm{~atm})$ to $80 \mathrm{MPa}$, that is enough for the most part of the industrial applications. In this context, it is simpler to use the Tait's empirical equation of state [74]:

$$
V_{m}(P, T)=V_{m}(0, T)\left\{1-C \ln \left[1+\left(P-P^{0}\right) / B(T)\right]\right\}
$$

where the coefficient $C$ is generally considered as an universal constant equal to 0,0894 [75]. The isothermal volume at zero pressure is given by:

$$
V_{m}(0, T)=V^{0}=V_{0} \exp (\alpha T)
$$

where $\alpha$ is the thermal expansion coefficient. The Tait's parameter $B(T)$ is homogeneous to a compressibility and is given by:

$$
B(T)=B_{0} \exp \left(-B_{1} T\right)
$$

The Equation (35) is equivalent to:

$$
\frac{1}{\rho_{m}}=\frac{\partial G_{m}}{\partial P}
$$


with:

$$
\begin{aligned}
& G_{m}=\left(\frac{1+C}{\rho_{m}^{0}}\right)\left(P-P^{0}\right) \\
& +\frac{C}{\rho_{m}^{0}}\left\{-B \ln \left(1+\frac{\left(P-P^{0}\right)}{B}\right)-\left(P-P^{0}\right) \ln \left(1+\frac{\left(P-P^{0}\right)}{B}\right)+B\right\}
\end{aligned}
$$

A second order Taylor's expansion of (39) gives:

$G_{m}=\left(\frac{1+C}{\rho_{m}^{0}}\right)\left(P-P^{0}\right)$

$+\frac{C}{\rho_{m}^{0}}\left\{B-\left(P-P^{0}\right)+\frac{\left(P-P^{0}\right)^{2}}{2 B}-\frac{\left(P-P^{0}\right)^{2}}{B}+\mathrm{O}\left(\left(\frac{\left(P-P^{0}\right)}{B}\right)^{3}\right)\right\}$

or,

$$
\begin{aligned}
G_{m} & =\frac{\left(P-P^{0}\right)}{\rho_{m}^{0}}+\frac{B C}{\rho_{m}^{0}} \\
& -\frac{C}{\rho_{m}^{0}}\left\{\frac{\left(P-P^{0}\right)^{2}}{2 B}+\mathrm{O}\left(\left(\frac{\left(P-P^{0}\right)}{B}\right)^{3}\right)\right\}
\end{aligned}
$$

The initial free enthalpy is defined for $P=P^{0}$ :

$$
G_{m}^{0}=G_{m}\left(P^{0}\right)=\frac{B C}{\rho_{m}^{0}}
$$

By holding only the terms of the same order in (32) and (33), some rearrangements give the following coupling equations:

$$
\underline{\underline{\sigma}}=\underline{\underline{\sigma}}^{0}+\underline{\underline{\underline{\underline{C_{0}}}}}: \underline{\underline{\varepsilon}} \underline{\varepsilon}_{e}-b\left(P-P^{0}\right) \underline{\underline{1}}
$$

with:

$$
\begin{gathered}
\underline{\underline{\underline{\underline{C_{0}}}}=\underline{\underline{\underline{C}}}-M b^{2} \underline{\underline{1}} \otimes \underline{\underline{1}}} \\
P=P^{0}+M\left[-b \varepsilon_{e}^{\mathrm{vol}}-\phi_{v p}\right]
\end{gathered}
$$

The tensor $\underline{\underline{\underline{C_{0}}}}$ is the tensor of elasticity of the solid phase. Because the material is isotropic, (24) yields:

$$
\phi_{v p}=\beta \operatorname{trace}\left(\underline{\underline{\varepsilon}}_{v p}\right)=\beta \varepsilon \varepsilon_{v p}^{\mathrm{vol}}
$$

and (44) becomes:

$$
P=P^{0}+M\left[-b \varepsilon_{e}^{\mathrm{vol}}-\beta \varepsilon_{v p}^{\mathrm{vol}}\right]
$$

The Equation (43) defines a notion of effective stress by:

$$
\underline{\underline{\sigma}}^{\prime}=\left(\underline{\underline{\sigma}}^{0}+b G_{m}^{0} \underline{\underline{1}}\right)+\underline{\underline{\underline{\underline{C_{0}}}}}: \underline{\underline{\varepsilon}}_{e}=\underline{\underline{\sigma}}+b P \underline{\underline{1}}
$$

This effective stress is the stress really applied to the solid skeleton. Through (46) and (47), the pressure $P$ in the free amorphous phase plays the role of a mechanical spring. Its evolution is given by the elastic and plastic volumetric strain of the material. This coupling indirectly shows that the volume strain of SCP can have an important effect on their global behavior.

\section{VISCOPLASTIC BEHAVIOR}

\subsection{Choice of the Yield Criterion}

In the proposed two-phase representation, the fluid phase (free amorphous) is supposed to behave, intrinsically, as a purely elastic material. The viscous and irreversible phenomena are due to physical mechanisms occurring in the solid part: crystalline sliding, deformation of the confined amorphous phase, molecular frictions, etc. Then, we suppose the existence of a yield surface in the stress space. It represents, at the macroscopic scale, the activation of all these mechanisms through the notion of plastic threshold and the notion of hardening. The exact shape of the yield surface can be determined only through multiaxial experiments on SCP. However, the presented experimental evidences (Section 1) combined to a two-phase interpretation of the microstructure let us compare SCP to soil-like materials. The dependence of the plastic yield on the mean stress, experimentally shown, leads to propose a general shape of the yield function:

$$
f=\sigma_{e q}-\mu_{s} f_{1}\left(p_{s}, r\right) \text { with } \sigma_{e q}=J_{2}^{1 / 2}\left(\underline{\underline{S}}_{\beta}\right)
$$

Equation (48) introduces the effective mean stress $p_{s}$ which is given, according to (26) and (47), by:

$$
\begin{aligned}
p_{s}=P_{\beta}=\frac{1}{3} \operatorname{trace}\left(\underline{\underline{\sigma}}_{\beta}^{\prime}\right) & =\frac{1}{3} \operatorname{trace}(\underline{\underline{\sigma}}+\beta P \underline{\underline{1}}) \\
& =\frac{1}{3} \operatorname{trace}\left(\underline{\underline{\sigma^{\prime}}}+(\beta-b) P \underline{\underline{1}}\right)
\end{aligned}
$$

or:

$$
p_{s}=\frac{1}{3} I_{1}\left(\underline{\underline{\sigma^{\prime}}}\right)+(\beta-b) P \underline{\underline{1}}
$$

The variable $p_{s}$ corresponds to the mean stress which really acts on the solid phase. The parameter $\mu_{s}$ is an internal friction parameter which governs the material sensitivity to the mean stress. Section 1 showed that the plastic flow of SCP is also associated to more or less irreversible expansion and contraction. On the other hand, the tests made on PA11 tend to show that the plastic yield before necking evolves towards an unique point, which corresponds to a stabilization of the volume strain. This is more significant in compression (Figs 10 and 11). Naturally, this is not necessarily valid for all the SCP. 
For soil-like or granular materials, it was shown that these phenomena correspond to a critical state, governed by the variation of the void ratio [76, 77]. One can consider that soils constitute typical materials for the study of two-phase systems. Indeed, for such materials, the fluid phase is very mobile and the viscoplastic behavior of the skeleton is directly accessible from experiments. By virtue of the previous experimental evidences, we think that it is possible to spread this analysis to SCP.

Taking into account the material isotropy, the hardening effects are described by using the following invariants of the viscoplastic strain:

$$
\varepsilon_{v p}^{d}=\sqrt{\frac{2}{3} \operatorname{dev}\left(\underline{\underline{\varepsilon}}_{v p}\right): \operatorname{dev}\left(\underline{\underline{\varepsilon}}_{v p}\right)} \text { and } \varepsilon_{v p}^{\mathrm{vol}}=\operatorname{trace}\left(\underline{\underline{\varepsilon}}_{v p}\right)
$$

Let us note $e$ the index of the space occupied by the free amorphous:

$$
e=\frac{\phi_{a}}{1-\phi_{a}}
$$

Then, a positive hardening "pressure" associated to the plastic volume strain is defined by:

$$
p_{c r}=p_{c r}^{0} \exp \left(\beta_{s}\left(e_{v p}-e_{v p}^{0}\right)\right)=p_{c r}^{0} \exp \left(\beta_{s} \varepsilon_{v p}^{\mathrm{vol}}\right)
$$

where $e_{v p}$ is the viscoplastic part of $e$ and $e_{v p}^{0}$ and $p_{c r}^{0}$ correspond to the reference state. This expression means that under the influence of an isotropic compaction of the material, the hardening pressure increases [76]. Let us note that for propellant-like materials, made of a polymer matrix filled with mineral inclusions, a similar expression was recently introduced to describe a volumetric plastic mechanism [78].

The material also undergoes hardening caused by the deviatoric plastic strain. This is an isotropic hardening which corresponds to an "expansion" of the yield surface. From a microscopic point of view, it results from the presence of obstacles which oppose to the growth of the plastic processes. The size of the elastic domain is:

$$
r=\frac{\varepsilon_{v p}^{d}+\varepsilon_{0}^{d}}{a+\varepsilon_{v p}^{d}+\varepsilon_{0}^{d}}
$$

where $\varepsilon_{0}^{d}$ corresponds to the initial plastic strain and $a$ is an isotropic hardening parameter.

Following (48), the yield surface is chosen similar to a generalized Cam-Clay surface:

$$
f=\sigma_{e q}-\mu_{s}\left(p_{s}-C_{s}\right)\left(1-b_{s} \ln \left[\frac{\left(P_{\beta}-C_{s}\right)}{p_{c r}}\right]\right) \cdot r
$$

Then, according to (53):

$$
f=\sigma_{e q}-\mu_{s}\left(p_{s}-C_{s}\right)\left(1-b_{s} \ln \left[\frac{\left(P_{\beta}-C_{s}\right)}{p_{c r}^{0}}\right]-b_{s} \beta_{s} \varepsilon_{v p}^{v o l}\right) \cdot r
$$

The parameter $b_{s}$ controls the "locking" of the yield surface in the space $\left(\sigma_{e q}, p_{s}=P_{\beta}\right)$. It defines a set of surfaces from the original Cam-Clay for $b_{s}=1$ to a Coulomb criterion for $b_{s}=0$.

The parameter $C_{s}$ is the cohesion of the material. It represents the critical effective mean stress in tension.

The expression (56) defines a yield surface the size of which is a function of the material hardening. The deviatoric part of the viscoplastic strain gives the size of the elastic domain through the internal state variable $r$. The parameter $\varepsilon_{0}^{d}$ corresponds to the initial size of this domain. The parameter $\beta_{s}$ controls the intensity of the volumetric hardening due to the viscoplastic volume strain. When $\varepsilon_{v p}^{0}$ is positive, the contribution of the term $b_{s} \beta_{s} \varepsilon_{v p}^{0}$ in (56) results in softening of the material. This decrease of the apparent stress is associated to the expansion which acts as a damaging mechanism. Conversely, a negative value of $\varepsilon_{v p}^{0}$ corresponds to a contraction and to a densification of the polymer. In that case, the hardening becomes positive and the stress increases during the deformation.

\subsection{Flow Rule}

Using the general viscoplastic formalism introduced by Perzyna [24], the intensity of the viscoplastic flow is determined by an "overstress" function, defined here by:

$$
Y=Y(f(r))=\sinh \left(\frac{f(r)}{K_{s}}\right)
$$

where $f$ is the yield surface (56) and $Y$ a characteristic function of the material and $K_{s}$ a parameter. The choice of a hyperbolic sine for the overstress function results from an analogy with Eyring's approach of the molecular viscosity. Here, the approach is macroscopic and it is simply a phenomenological transcription of this theory. Furthermore, the experience of the developed model shows us that this expression is adequate to describe the long time relaxation of $\mathrm{PVF}_{2}$.

The relation $f=0$ introduces a static surface which corresponds to very slow loading. For example, it is reached at the end of relaxation tests.

The flow rule is written as:

$$
\underline{\underline{\varepsilon}}_{v p}=\frac{1}{\eta} Y(f(r)) \Lambda\left(\underline{\underline{S}}_{\beta}\right)
$$

with:

$$
\Lambda\left(\underline{\underline{S}}_{\beta}\right)=\frac{\sqrt{3}}{2} \frac{\underline{S}_{\beta}}{\sigma_{e q}}+\frac{\alpha_{s}}{3}\left(\mu_{s}-\frac{\sigma_{e q}}{\left(P_{\beta}-C_{s}\right)}\right) \underline{\underline{1}}
$$

which from (51) yields:

$$
\dot{\varepsilon}_{v p}^{d}=\frac{1}{\eta} Y(f(r))
$$


and:

$$
\dot{\varepsilon}_{v p}^{\mathrm{vol}}=\frac{1}{\eta} Y(f(r)) \alpha_{s}\left(\mu_{s}-\frac{\sigma_{e q}}{\left(P_{\beta}-C_{s}\right)}\right)
$$

The parameter $\eta$ is a viscosity parameter. It can be compared to a relaxation time. The material parameter $\alpha_{s}$ controls the intensity of the volume strain.

Figure 23 shows the effect of the parameter $b_{s}$ on the yield surface. The nonassociated flow rule (58)-(60) is also represented. Equation (60) introduces a critical state line which separates the $\left(\sigma_{e q}, \mathrm{p}_{s}\right)$ space into a part associated to a dilatant flow (expansion) and a part associated to a contractant flow (compaction).

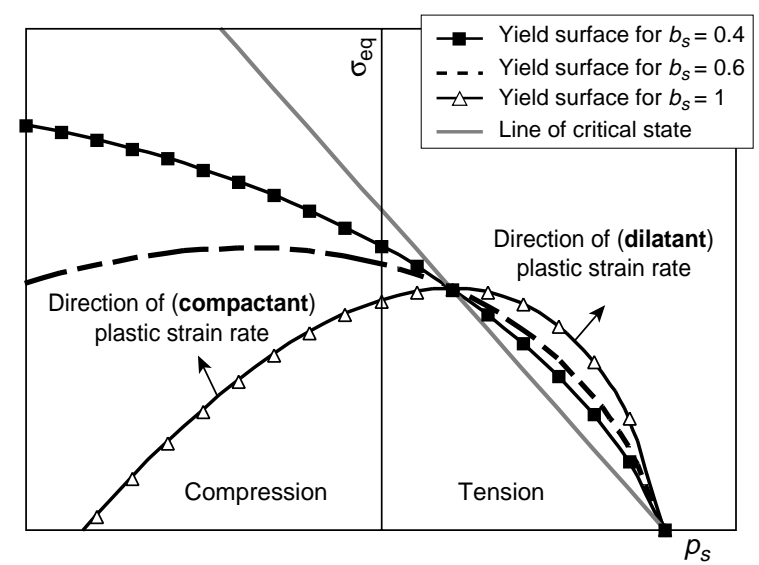

Figure 23

Illustration of the nonassociated flow rule for several values of $b_{s}$, showing the yield surfaces associated to the hard solid phase (crystallites and confined amorphous).

\subsection{Thermodynamic Considerations}

The considered viscoplastic behavior is non standard. The hardening evolution and the flow rules can be deduced from the precedent thermodynamic framework by using nonassociated potentials [39].

The flow rule (58) may be deduced from a pseudo potential $\Phi_{1}$ depending on the strain rate, by using the following complementary law:

$$
\underline{\underline{\sigma}}_{\beta}=\frac{\partial \Phi_{1}}{\partial \underline{\underline{\varepsilon}}_{v p}}
$$

where
Let us now consider the following internal state variables:

$\gamma_{i}=\left\{\gamma^{d}, \gamma^{\text {vol }}\right\}$ associated to the affinities: $A_{i}=\left\{r, p_{c r}\right\}(63)$

The state equations are deduced from a specific choice of the stored energy $U$ introduced in the free energy (31):

$$
\begin{aligned}
& U\left(\gamma^{d}, \gamma^{\mathrm{vol}}\right)= \\
& \left(\gamma^{d}+\gamma_{0}\right)-a \ln \left(\left(\gamma^{d}+\gamma_{0}\right)+a\right)+\frac{p_{c r}^{0}}{\beta_{s}} \exp \left(\beta_{s} \gamma^{\mathrm{vol}}\right)
\end{aligned}
$$

Then, Equations (34) and (64) give:

$$
r=\frac{\gamma^{d}+\gamma_{0}}{a+\gamma^{d}+\gamma_{0}}
$$

and

$$
p_{c r}=p_{c r}^{0} \exp \left(\beta_{s} \gamma^{\mathrm{vol}}\right)
$$

By analogy with the theory of plasticity, complementary laws can be given for internal state variables:

$$
\left(\dot{\gamma}^{d} ; \dot{\gamma}^{\mathrm{vol}}\right)=\frac{1}{\eta} Y(f)\left(\frac{\partial \Phi_{\gamma}}{\partial r} ; \frac{\partial \Phi_{\gamma}}{\partial p_{c r}}\right)
$$

with the following choice for nonassociated potential:

$$
\Phi_{\gamma}=r+\alpha_{s}\left(\mu_{s}-\frac{\sigma_{e q}}{P_{\beta}-C_{s}}\right) p_{c r}
$$

By using (67) and (68), the evolution of the internal state variables is:

$$
\begin{gathered}
\dot{\gamma}^{d}=\frac{1}{\eta} Y(f) \\
\dot{\gamma}^{\mathrm{vol}}=\frac{1}{\eta} Y(f) \alpha_{s}\left(\mu_{s}-\frac{\sigma_{e q}}{P_{\beta}-C_{s}}\right) .
\end{gathered}
$$

Then, thanks to (59) and (60), the internal state variables are identified as:

$$
\dot{\gamma}^{d}=\dot{\varepsilon}_{v p}^{d} \text { and } \dot{\gamma}^{\mathrm{vol}}=\dot{\varepsilon}_{v p}^{\mathrm{vol}}
$$

\section{VALIDATION OF THE MODEL}

The constitutive equations are recapitulated hereafter:

$$
\begin{gathered}
\underline{\underline{\sigma}}=\underline{\underline{\sigma^{\prime}}}-b P \underline{\underline{1}} \\
\underline{\underline{\sigma}}_{\beta}^{\prime}=\underline{\underline{\sigma^{\prime}}}+(\beta-b) P \underline{\underline{1}}
\end{gathered}
$$

$$
\Phi_{1}\left(\underline{\underline{\varepsilon}}_{v p}\right)=\frac{2}{\sqrt{3}} \sigma_{e q}\left[\eta Y(f) \underline{\underline{\varepsilon}}_{v p}: \dot{\underline{\varepsilon}}_{v p}-\frac{\alpha_{s}}{3}\left(\mu_{s}-\frac{\sigma_{e q}}{\left(P_{\beta}-C_{s}\right)}\right) \underline{1}: \dot{\underline{\varepsilon}}_{v p}\right]+P_{\beta} 1: \dot{\underline{\varepsilon}}_{v p}
$$




$$
\begin{gathered}
P=P^{0}+M\left[-b \varepsilon_{e}^{\mathrm{vol}}-\beta \varepsilon \varepsilon_{v p}^{\mathrm{vol}}\right] \\
\underline{\underline{S}}_{\beta}=\underline{\underline{\operatorname{dev}}}\left(\underline{\underline{\sigma}}_{\beta}^{\prime}\right) \text { and } P_{\beta}=\frac{1}{3} \operatorname{trace}\left(\underline{\underline{\sigma}}_{\beta}^{\prime}\right)
\end{gathered}
$$

$$
f=\sigma_{e q}-\mu_{s}\left(P_{\beta}-C_{s}\right)\left(1-b_{s} \ln \left[\frac{\left(P_{\beta}-C_{s}\right)}{p_{c r}^{0}}\right]-b_{s} \beta_{s} \varepsilon_{v p}^{\mathrm{vol}}\right) \cdot r
$$

with:

$$
\begin{gathered}
\sigma_{e q}=J_{2}^{1 / 2}\left(\underline{\underline{S}}_{\beta}\right) \text { and } r=\frac{\varepsilon_{v p}^{d}+\varepsilon_{0}}{a+\varepsilon_{v p}^{d}+\varepsilon_{0}} \\
\underline{\underline{\varepsilon}}_{v p}=\frac{1}{\eta} Y(f(r)) \Lambda\left({\underline{\underline{\sigma^{\prime}}}}_{\beta}\right)
\end{gathered}
$$

with:

$$
\Lambda\left({\underline{\underline{\sigma^{\prime}}}}_{\beta}\right)=\frac{\sqrt{3}}{2} \frac{\underline{\underline{S}}_{\beta}}{\sigma_{e q}}+\frac{\alpha_{s}}{3}\left(\mu_{s}-\frac{\sigma_{e q}}{P_{\beta}-C_{s}}\right) \underline{\underline{1}}
$$

which leads to:

$$
\begin{aligned}
\dot{\varepsilon}_{v p}^{\mathrm{vol}} & =\frac{1}{\eta} Y(f(r)) \operatorname{trace}\left(\Lambda\left({\underline{\underline{\sigma^{\prime}}}}_{\beta}\right)\right) \\
= & \frac{1}{\eta} Y(f(r)) \alpha_{s}\left(\mu_{s}-\frac{\sigma_{e q}}{P_{\beta}-C_{s}}\right) \\
\dot{\varepsilon}_{v p}^{d}=\frac{1}{\eta} & Y(f(r)) J^{2}\left(\operatorname{dev}\left(\Lambda\left({\underline{\underline{\sigma^{\prime}}}}_{\beta}\right)\right)\right)=\frac{1}{\eta} \mathrm{Y}(f(r))
\end{aligned}
$$

Fifteen material parameters must be identified to run the model. The coupling parameters of Equations (71)-(73) are estimated from considerations on the ERV and from the quantities of free amorphous and confined amorphous which are obtained from physicochemical identification. They are parameters associated to the compressibility of the solid and fluid phases. Furthermore, thermodynamic considerations allow to limit these parameters and to not deviate from physically coherent solutions. The viscosity parameters of Equations (75) and (76) are estimated from tensile tests performed at various loading rates. The ability to reproduce the material response, on this monotonous experiment, already establishes a first validation of the model. A complete validation needs simulate other loading paths (creeping, relaxation, etc.) with the same set of parameters. The estimation of the plastic parameters of the yield surface, needs theoretically several tests associated to multi-axial loading paths. However, practically, a first estimation is obtained from usual tensile tests. A validation of this first choice consist to compare the predictive response of the model with homogeneous tests in compression, for example, or with structural non homogeneous tests induce multi-axial stress state. A complete procedure of validation is examined in [1].

Figures 24 and 25 show the model ability to reproduce tensile tests on a $\mathrm{PVF}_{2}$, and, particularly to predict the volume strain. The crystalline index of $\mathrm{PVF}_{2}$ is $48 \%$. At room temperature, most of the amorphous is in a confined state. Then, the proportion of free amorphous is rather weak and the model predict an important volumetric expansion
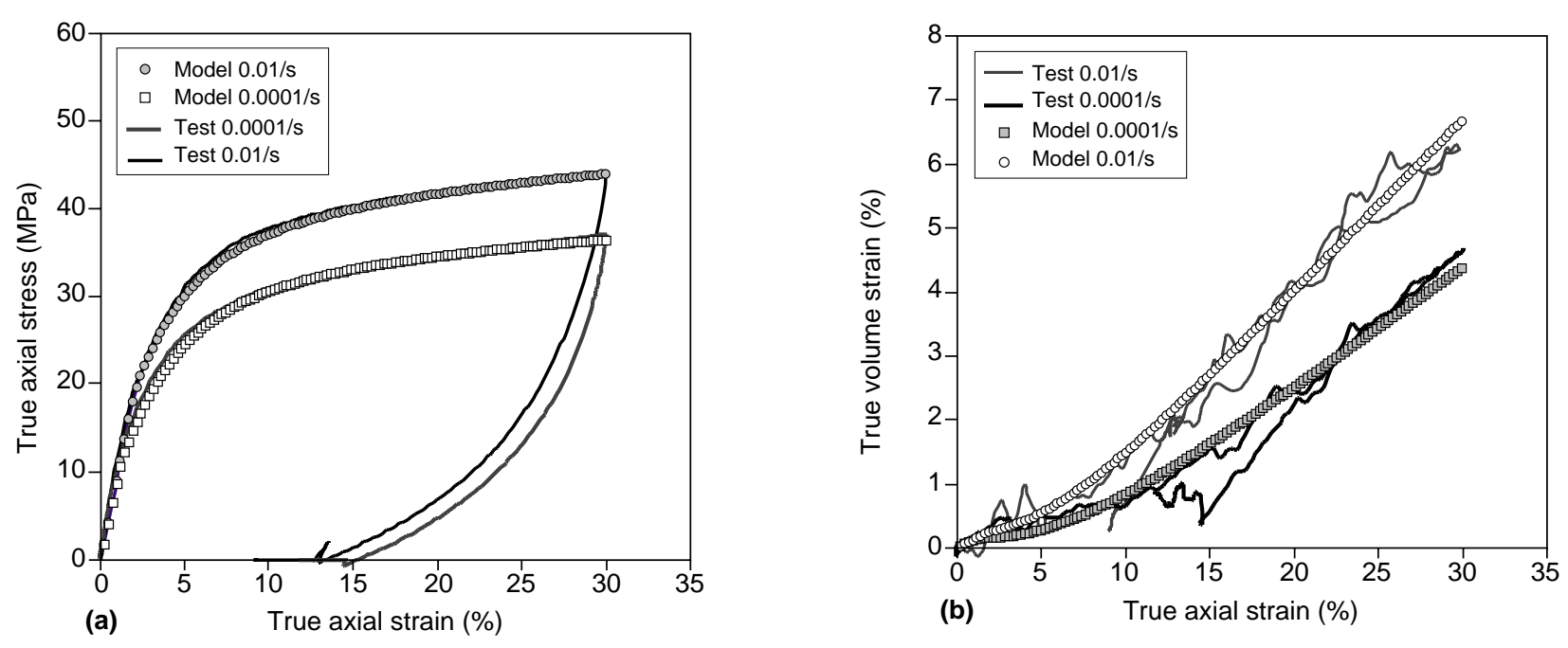

Figure 24

$\mathrm{PVF}_{2}$. Comparison of tensile tests at $10^{-3} / \mathrm{s}$ and $10^{-4} / \mathrm{s}$ and model responses (a) axial stress/strain curves and (b) volume strain/axial strain curves. 


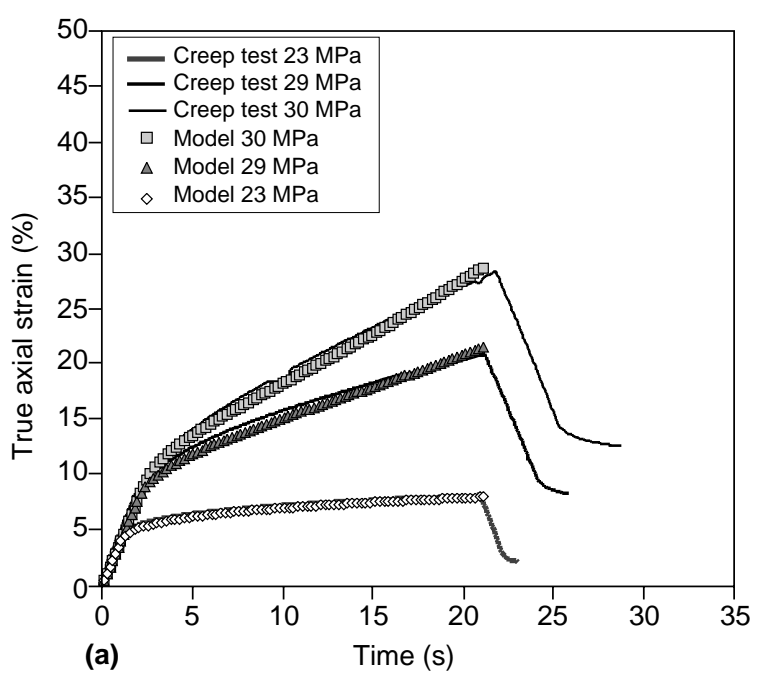

Figure 25

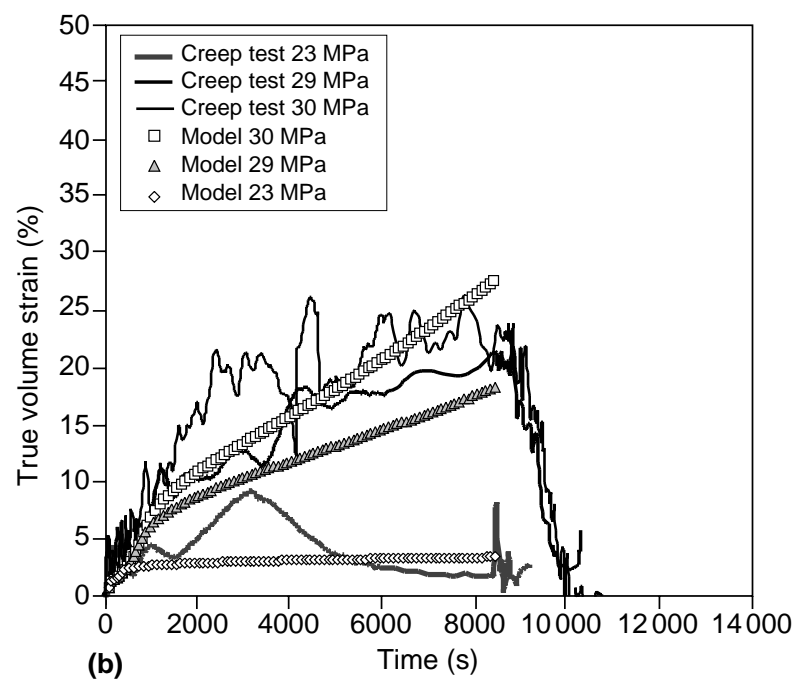

$\mathrm{PVF}_{2}$. Comparison of creep test and model response (a) axial strain and (b) volume strain.

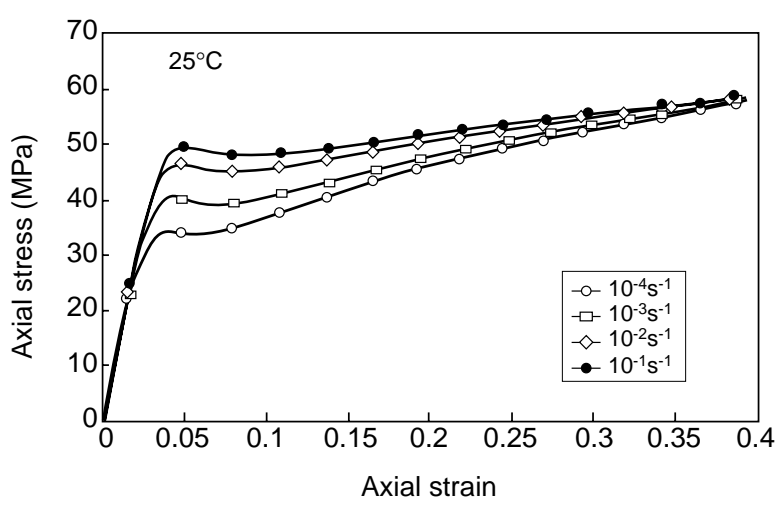

Figure 26

PA11. Stress/strain for four tensile tests at different loading rates, at $25^{\circ} \mathrm{C}$ (after [79]).

according to the experimental results. One can also notice that the proposed approach is efficient for both the short time response (effect of the loading rate) and the long time response (creeping effect).

The PA11 behavior is radically different compared to that of $\mathrm{PVF}_{2}$. Figure 26 shows the evolution of the axial stress versus the axial strain for three tensile tests conducted at different strain rates. Two thresholds are obtained, one characterized by the beginning of the plastic domain, towards $4 \%$ of axial strain, and the other associated to a stabilization of the axial stress. This convergence of the axial stress happens towards $30 \%$ of axial strain, from which necking appears in the samples.

The PA11 crystalline index is low (20\%). Considering the deuteration results of Figure 20, one can consider that at room temperature, at least $50 \%$ of the amorphous phase is not perturbed by the presence of the crystallites. Then, the free amorphous proportion is about $40 \%$. Here, the soft phase is going to play an important role in the behavior of the material through the coupling Equations (71)-(73). Figure 27 shows the prediction of the model compared to tests performed on a PA11, dried three days at $80^{\circ} \mathrm{C}$ under vacuum. One can note the good agreement of curves and the model ability to reproduce the phenomena of double thresholds. The volume strain of PA11 is also correctly predicted as it is shown on Figure 28, where the experiment reveals a contraction tendency.

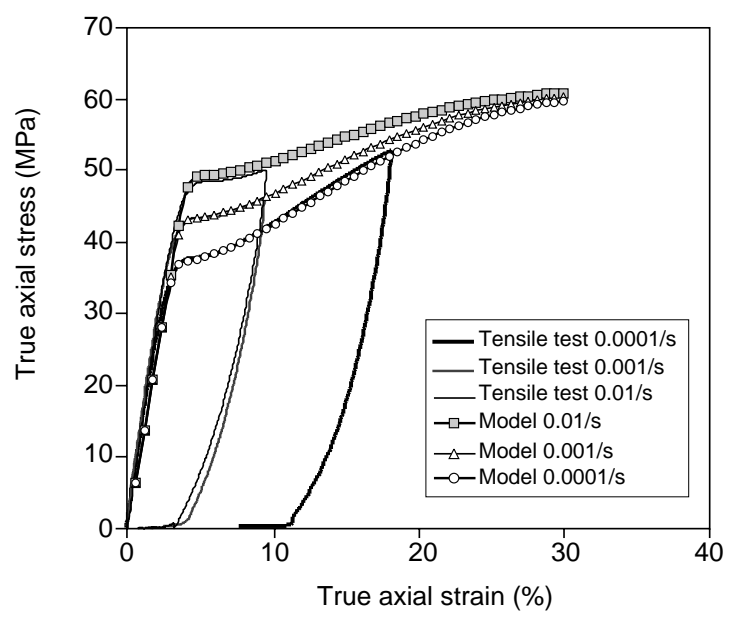

Figure 27

Comparison model/experiment at $25^{\circ} \mathrm{C}$, for tensile tests performed on dried PA1 1 at $80^{\circ} \mathrm{C}$, showing the model capability to reproduce the double plastic thresholds. 

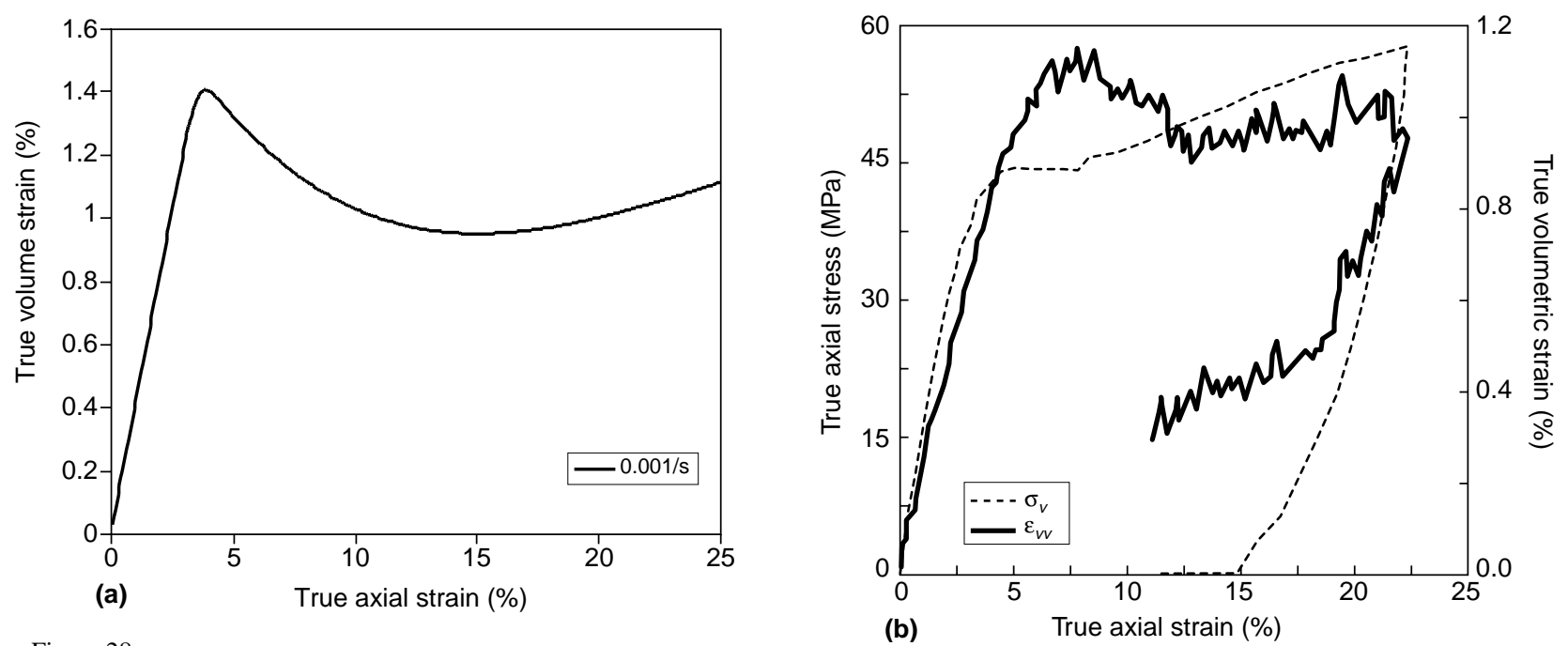

Figure 28

(b)

PA11. Volumetric effects obtained in a tensile test performed at room temperature and at a strain rate of $10^{-3} / \mathrm{s}$ (a) prediction of the model (b) measures by laser extensometry (after [54]).
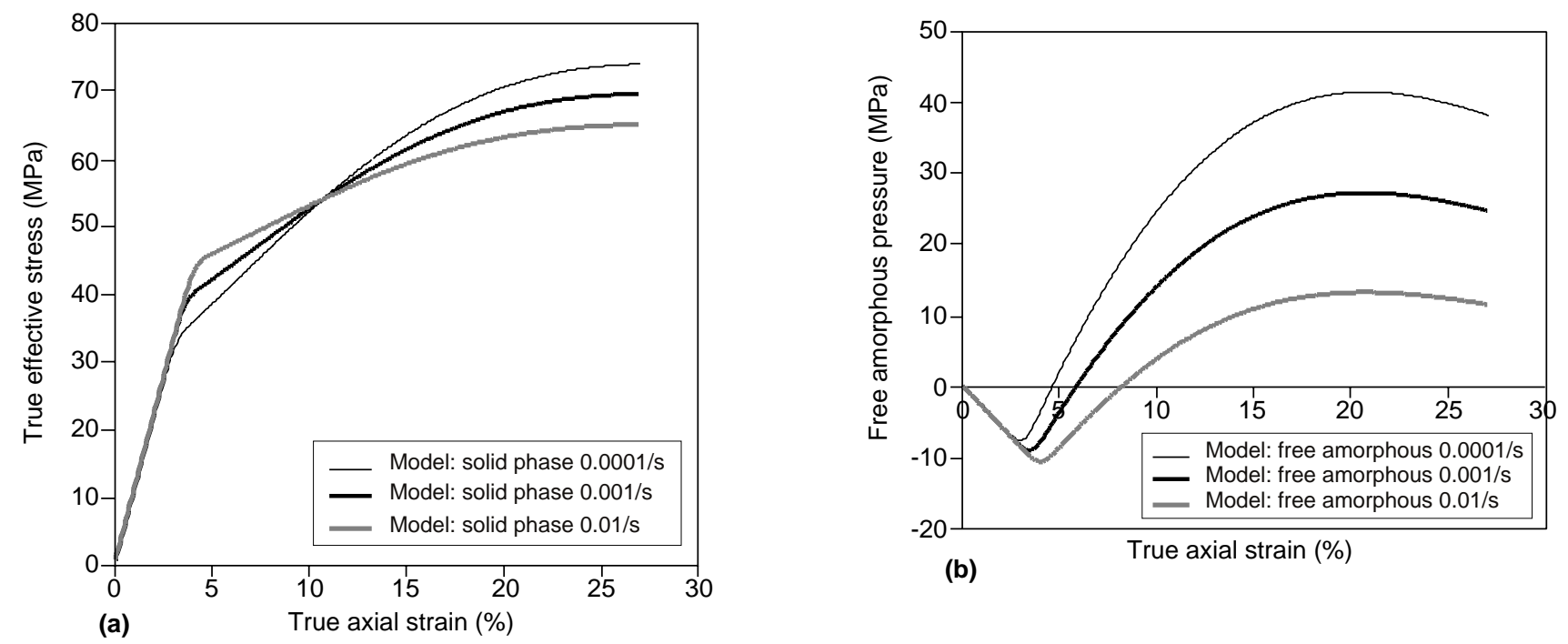

Figure 29

PA11. Contribution of the solid and soft phases during a tensile test (a) stress/strain curves for the solid phase (b) internal pressure of free amorphous/axial strain.

Figure 29 gives the contribution of each phase to the total stress (or apparent stress), for three tensile tests at increasing loading rates. Figure 29a shows the evolution of the effective stress undergone by the crystalline part and the confined amorphous. The first threshold is governed essentially by viscous and thermally activated mechanisms, and increases with the loading rate. On the other hand, the second threshold corresponds to the progressive saturation of the material plastic hardening. One can notice that this second threshold decreases with the loading rate. This effect is due to the contractant evolution of the plastic volume in the yield surface $f(75)$.

Figure 29b draws the evolution of the pressure in free amorphous. Here, this phase reacts as a mechanical spring and undergoes the effects of the material volume change. At the beginning of the test, the volume strain is essentially positive due to the elastic expansion according to Hooke's law and the pressure in the amorphous is negative. When the first plastic threshold is reached, the plastic contraction infers a compression on free amorphous and the internal pressure in 
this phase begins increasing towards positive values. So, the convergence of the apparent stress towards an unique threshold, results, in this interpretation of the material, from the combination of the decrease of the effective stress in the solid phase, with the increase of the fluid pressure in the free amorphous phase.

\section{CONCLUSION}

A model of mechanical behavior was developed from a twophase interpretation of the microstructure of SCP at a mesoscopic scale. This interpretation is based on the partition of the material into a solid or "hard" phase, and a "soft" or fluid phase, experimentally justified. In parallel, experimental evidences were gathered so as to underline the influence of the mean stress and the volume strain on the SCP global mechanical behavior. These two aspects, microstructural and phenomenological, lead to develop an approach close to methods well-tried in geomechanics. The model is physically based on the thermodynamics of open systems. The conservation equations allow to derive, in a coherent way, an interaction equation between both phases. This equation introduces a notion of effective stress applied to the solid part of the material. This phase is supposed to be at the origin of the irreversible and viscoplastic evolution of the material. A specific yield surface and the Perzyna formalism are used to describe the viscoplastic deformation. The accent is put on the description of the phenomena of hardening, by introducing specific mechanisms associated to the deviatoric and volumetric strain.

The obtained model is validated in tension on $\mathrm{PVF}_{2}$ and on PA11. The two-phase nature allows to overcome apparent contradictions of the mechanical responses of these two polymers. In the case of $\mathrm{PVF}_{2}$, the response to the short time loading and to the long time loading are correctly reproduced, as well as the plastic expansion arising during the irreversible evolutions of the material. The proportion of amorphous or of free amorphous plays a significant role in the case of PA11 and allows to give a mechanical interpretation of the presence of the double thresholds, connected also to the notion of volume contraction.

The development of the model in a general framework of thermodynamics constitutes interesting bases for taking into account complex loading and for introducing thermal, mechanical and physicochemical couplings, for the study of the long term behavior of SCP.

\section{ACKNOWLEDGMENTS}

The authors would like to thank Jean Louis Gacougnolle and Jacques Parisot from the Laboratoire de Mécanique et Physique des Matériaux - CNRS UMR 6617, France, for fruitful discussions and for the realization of tests shown on Figures 24 and 25, and IFP colleagues for valuable contributions and discussions: Patrick Bourguelat for the realization of tension tests on $\mathrm{PVF}_{2}$ and on PA11, MarieHélène Klopffer, Bernard Dewimille, Jacques Jarrin for fruitful discussions, Élisabeth Bemer, Jacques Daligot, Guy Pignard, Olivier Vincké for the compression tests on PA11, Élisabeth Rosenberg for SEM analysis on PA11 and Nathalie Zanier for helpful discussions on FT-NIR analysis.

\section{REFERENCES}

1 Meimon, Y. (2000) Some Challenges of the Development of Constitutive Equations of Semicrystalline Polymers for Structural Calculations.Oil \& Gas Science and Technology, $\mathbf{5 5}, 6,649-659$.

2 Gaillard-Devaux, E. (1995) Rupture du polyéthylène en température par décompression de méthane. Thèse, École nationale supérieure des mines de Paris.

3 Bauwens-Crowet, C., Bauwens, J. and Homes, G. (1969) Tensile Yield-Stress Behavior of Glassy Polymers. J. Polym. Sci. - Part A-2, 7, 735-742.

4 Sternstein, S.S., Ongchin, L. and Silverman, A. (1968) Inhomogeneous Deformation and Yielding of Glasslike High Polymers. Appl. Polym. Symp., 7 175-199

5 Bahadur, S. (1973) Strain Hardening Equation and the Prediction of Tensile Strength of Rolled Polymers. Polym. Eng. Sci., 13, 266-272.

6 Bowden, P.B. and Young, R.J. (1974) Deformation Mechanism in Crystalline polymers, J. Mater. Sci., 9, 20342051.

7 Haudin, J.M. (1982) in Plastic Deformation of Amorphous and Semicrystalline Materials, B. Escaig and C. G'Sell, eds., Les éditions de physique, Les Ullis Cedex, France, 291-311.

8 Argon, A.S. (1973) A Theory for the Low-Tempature Plastic Deformation of Glassy Polymers. Phil. Mag., 28, 839-865.

9 Escaig, B. (1984) A Metallurgical Approach to the Pre-yield an yield Behavior of Glassy Polymers, Polym. Eng. Sci., 24, $10,737-749$.

10 Perez, J. (1992) Physique et mécanique des polymères amorphes, Lavoisier, Tec et Doc, Paris, France.

11 G'Sell, C. (1986) Plastic Deformation of Glassy Polymers: Constitutive Equations and Macromolecular Mechanisms, in Strength of Metals and Alloys, Ed. H.J. McQueen et al., 3, Pergamon Press, Oxford, UK, 1946-1982.

12 Boyce, M.C. and Arruda, E.M. (1990) An Experimental and Analytical Investigation of the Large Strain Compressive and Tensile Response of Glassy Polymers. Polym. Eng. Sci., 30, 1288-1298.

13 Amadeo, J. and Lee, D. (1992) Modelling the Uniaxial Rate Temperature Dependent Behaviour of Amorphous and Semicrystalline Polymers. Polymer Engineering and Science, 32, 16, 1055-1065.

14 G'Sell, C. and Jonas, J.J. (1979) Determination of the Plastic Behaviour of Solid Polymers at Constant True Strain Rate. J. Mater. Sci., 14, 583-591.

15 G'Sell, C. and Jonas, J.J. (1981) Yield and Transient Effects During the Plastic Deformation of Solid Polymers. J. Mater. Sci., 16, 1956-1974. 
16 Eyring, H., Glasstone, S. and Laidler, K.J. (1941) The Theory of Rate Processes: the Kinetics of Chemical Reactions, Viscosity, Diffusion and Electrochemical Phenomena, Mc Graw-Hill Book Cy., New York.

17 Treloar, L.R.G. (1995) The Physics of Rubber Elasticity, $3^{\text {rd }}$ ed., Clarendon, Oxford.

18 Boyce, M.C., Parks, D.M. and Argon, A.S. (1988) Large Inelastic Deformation of Glassy Polymers - Part I: Rate Dependent Constitutive Model. Mech. Mater., 7, 15-33.

19 Wu, P.D. and Van der Giessen, E. (1993) On Improved NonGaussian Network Models for Rubber Elasticity and their Implications to Orientation Hardening in Glassy Polymers. $J$. Mech. Phys. Solids, 41, 427-456.

20 O'Dowd, N.P. and Knauss, W.G. (1995) Time Dependent Large Principal Deformation of Polymers. J. Mech. Phys. Solids, 43, 771-792.

21 Krempl, E. (1979) Viscoplasticity Based on Total Strain. The Modelling of Creep with Special Considerations of Initial Strain and Aging. Trans. ASME, J. Eng. Mater. Tech., 101, 380-386.

22 Kitagawa, M., Mori, T. et Matsutani, T. (1989) RateDependant Nonlinear Constitutive Equation of Polypropylene. J. Polym. Sci. - Part B: Polymer Physics, 27, 85-95.

23 Brusselle-Dupend, N. (2000) Comportement viscoélastoplastique d'un polymère semi-cristallin avant la striction : caractérisation expérimentale et modélisation phénoménologique. Thèse, université technologique de Compiègne.

24 Perzyna, P. (1963) The Constitutive Equations for Rate Sensitive Plastic Materials. Quartely of Applied Mathematics, 20, 321-332.

25 Lemaitre, J. et Chaboche, J.L. (1985) Mécanique des matériaux solides, Dunod, Paris.

26 Cunat, C. (2001) The DNLR Approach and Relaxation Phenomena. Part I - Historical Account and DNLR Formalism. Mechanics of Time-Dependent Materials, 5, 3965.

27 Cunat, C. (1991) A Thermodynamic Theory of Relaxation Based on a Distribution of Nonlinear Processes. J. of Non Crystalline Solids, 131/133, 196-199.

28 Dahoun, A. (1992) Comportement plastique et textures de déformation des polymères semi-cristallins en traction uniaxiale et en cisaillement simple. Thèse, INPL.

29 Dahoun, A., Aboulfaraj, M., G'Sell, C., Molinari, A. and Canova, G.R. (1995) Plastic Behavior and Deformation Textures of Poly(Etherether Ketone) Under Uniaxial Tension and Simple Shear. Polym. Eng. Sci., 35, 4, 317-330.

30 G'Sell, C., Dahoun, A. (1994) Evolution of Microstrusture in Semicrystalline Polymers under Large Plastic Deformation. Materials Science and Engineering, A175, 183-199.

31 Lee, B., Parks, D. and Ahzi, S. (1993) Micromechanical Modeling of Large Plastic Deformation and Texture Evolution in Semicrystalline Polymers. J. Mech. Phys. Solids, 41, 1651-1687.

32 Chen, M.X., Zheng, Q.S. and Yang, W. (1996) A Micromechanical Model of Texture Induced Orthotropy in Plane Crystalline Polymers. J. Mech. Phys. Solids, 44, 157178.

33 Argon, A.S. (1997) Morphological Mechanisms and Kinetics of Large-Strain Plastic Deformation and Evolution of Texture in Semicrystalline Polymers. Journal of ComputerAided Materials Design, 4, 75-98.
34 Berveiller, M. and Zaoui, A. (1995) Modélisation du comportement mécanique des solides microhétérogènes, in Introduction à la mécanique des polymères, C. G'Sell et J.M. Haudin, Institut national polytechnique de Lorraine, 225-249.

35 Paquin, A. (1998) Modélisation micromécanique du comportement élastoviscoplastique des matériaux hétérogènes. Thèse, université de Metz.

36 Rougier, Y. (1994) Étude du comportement sous irradiation : modélisation micromécanique de l'élastoviscoplasticité. Thèse, École polytechnique.

37 Meimon, Y. and Cangémi, L. (1999) Polymères semicristallins à usage structural : microstructures, comportement mécanique. Proc. of Colloque national mecamat, 79-84.

38 Coussy, O., (1989) Thermodynamics of Saturated Porous Solids in Finite Deformation, Eur. J.; Mech. A/Solids, 8, 1-14.

39 Coussy, O. (1991) Mécanique des milieux poreux, Technip, Paris.

40 Coussy, O. (1995) Mechanics of Porous Continua, Wiley, New York.

41 Prigogine, I. (1967) Thermodynamics of Irreversible Processes. $3^{\text {rd }}$ ed., Interscience, New York.

42 Biot, M.A. (1972) Theory of Finite Deformations of Porous Solids. Indiana University Mathematics Journal, 21, 7, 597620.

43 G'Sell, C. (1995) Lois de comportement mécanique des polymères solides, in Introduction à la mécanique des polymères, C. G'Sell et J.M. Haudin, Institut national polytechnique de Lorraine, 141-168.

44 Chaboche, J.L. (1995) Formalisme général des lois de comportement : applications aux métaux et polymères, in Introduction à la mécanique des polymères, C. G'Sell et J.M. Haudin, Institut national polytechnique de Lorraine, 119-140.

45 G'Sell, C. (1995) Lois de comportement mécanique des polymères solides, in Introduction à la mécanique des polymères, C. G'Sell et J.M. Haudin, Institut national polytechnique de Lorraine, 141-168.

46 Pae, K.D. and Mears, D.R. (1968) The Effects of High Pressure on Mechanical Behavior and Properties of Polytetrafluoroethylene and Polyethylene. Polymer Letters, 6, 269-273.

47 Sardar, D., Radcliffe, S.V. and Baer, E. (1968) Effects of High Hydrostatic Pressure on the Mechanical Behavior of a Crystalline Polymer-Polyoxymethylene. Polymer Eng. Sci., 8, 290-301

48 Quinson, R., Perez, J., Rink, M. and Pavan, A. (1997) Yield Criteria for Amorphous Glassy Polymers. J. Materials Science, 32, 1371-1379.

49 Castagnet, S. (1998) Comportement mécanique du PVDF : compétition entre cavitation et écoulement visqueux. Thèse, université de Poitiers.

50 Whitney, W. and Andrews, R.D. (1967) Yielding of Glassy Polymers: Volume Effects. J. Polym. Sci. - Part C: Polymer Letters, 16, 2981-2990.

51 Kitagawa, M. and Yoneyama, T. (1988) Plastic Dilatation Due to Compression in Polymer Solids. J. Polym. Sci. - Part C: Polymer Letters, 26, 206-212.

52 Gaucher-Miri, V., Depecker, C. et Séguéla, R. (1997) Reversible Strain-Induced Order in the Amorphous Phase of Low-Density Ethylene/Butene Copolymer. J. of Polym. Sci. Part B: Polymer Physics, 35, 2151-2159.

53 Gaucher-Miri, V. (1995) Étude de la plasticité des polyéthylènes en traction uniaxiale. Thèse, université des sciences et technologies de Lille. 
54 Marchal, K. (1996) Influence du chemin de chargement sur le comportement du polyamide 11 autour de la transition vitreuse. Thèse, université de Poitiers.

55 Castagnet, L., Gacougnolle, J.L. and Dang, P. (2000) Correlation between Macroscopical Viscoelastic Behaviour and Micromechanisms in Strained $\alpha$ Polyvinylidene Fluoride $\left(\mathrm{PVF}_{2}\right)$. Materials Science and Engineering, A276, 152-159.

56 Quatravaux, T., Elkoun, S.,G'Sell, C., Cangémi, L. and Meimon, Y. On the Experimental Characterization of Volume Strain of Poly(Vinylidene Fluoride) in the Region of Homogeneous Plastic Deformation. Submitted to Journal of Polymer Science.

57 Elkoun, S., G'Sell, C., Cangémi, L. and Meimon, Y. Characterization of Volume Strain of Poly(Vinylidene Fluoride) under Creep Test. Submitted to Journal of Polymer Science.

58 Haudin, J.M. (1995) Structures et morphologies des polymères semi-cristallins, in Introduction à la mécanique des polymères, C. G'Sell et J.M. Haudin, Institut national polytechnique de Lorraine, 97-115.

59 Oudet, C. (1994) Polymères. Structure et propriétés. Introduction, Masson, Paris.

60 Magill, J.H. (2001) Review Spherulites: A Personal Perspective. J. Mater. Sci., 36, 3143-3164.

61 Struik, L.C.E. (1978) Physical Aging in Amorphous Polymers and Other Materials, Elsevier, Amsterdam.

62 Struik, L.C.E. (1987) The Mechanical and Physical Ageing of Semicrystalline Polymers: 1. Polymer, 28, 1521-1533.

63 Smit, P.P.A. (1966) The Glass Transition in Carbon Black Reinforced Rubber. Rheol. Acta, 5, 277-283.

64 Kraus, G. (1971) Reinforcement of Elastomers by Carbon Black. Adv. Polym. Sci., 8, 155-237.

65 Wu, P., Siesler, H.W., Dal Maso, F. and Zanier, N. (1998) Rheo-optical Fourier-Transform NIR Spectroscopy of Polyamide 11. Analusis Magazine, 26, 4, 61-64.

66 Klopffer, M.H. and Flaconnèche, B. (2001) Transport Properties of Gases in Polymers: Bibliographic Review. Oil \& Gas Science and Technology - Rev. IFP, 56, 3, 223-244.
67 Dal Maso, F., Barré, L., Espinat, D., Jarrin, J. and Boscher, Y. (1994) Simulation de la croissance de sphérolites de polymère et de spectres de diffusion centrale des rayons $\mathrm{X}$. Revue de l'Institut français du pétrole, 49, 4, 380-384.

68 Takayanagi, T. (1970) Viscoelastic Behavior of Crystalline Polymers. Fourth International Congress of Rheology, 161187.

69 Weitsman, Y. (1987) Stress Assisted Diffusion in Elastic and Viscoelastic Materials. J. Mech. Phys. Solids, 35, 1, 73-93.

70 Curro, J.G. (1974) Polymeric Equations of State. J. Macromol. Sci. - Rev. Macromol. Chem., C11, 2, 321-366.

71 Sanchez, I.C. and Lacombe, R.H.(1978) Statistical Thermodynamics of Polymer Solutions. Macromolecules, 11, 6, 1145-1156.

72 Simha, R. (1997) Configurational Thermodynamics of the Liquid and Glassy Polymeric states. Macromolecules, 10, 5, 1025-1030.

73 Dee, G.T. and Walsh, D.J. (1988) Equations of State for Polymer Liquids. Macromolecules, 21, 811-815.

74 Tait, P.G. (1888) Phys. Chem., $2,1$.

75 Nanda, V.S. and Simha, R. (1964) Equation of State of Polymer Liquids and Glasses at Elevated Pressures. J. Chem. Phys., 41, 12, 3870-3878.

76 Schofield, A.N. and Wroth, C.P. (1968) Critical State Soil Mechanics, Mc Graw Hill.

77 Roscoe, K.H., Schofield, A.N. and Wroth, C.P. (1968) On the Yielding of Soils. Géotechnique, 9, 71.

78 Trumel, H., Dragon, A., Fanget, A. and Lambert, P. (2001) A Constitutive Model for the Dynamic and High-Pressure behaviour of a Propellant-Like Material: Part II: Model Development and Application. Int. J. Numer. Anal. Meth. Geomech., 25, 581-603.

79 Bélec, L. (1995) Mise en évidence de la contribution des phases amorphe et cristalline dans la déformation du polyamide 11 et du polyamide 12 autour de la transition vitreuse. Thèse, université de Poitiers.

Final manuscript received in December 2001 